\title{
Highly Buoyant Bent-Over Plumes In a Boundary Layer
}

\author{
Ali Tohidi ${ }^{\mathrm{a}, *}$, Nigel B. Kaye ${ }^{\mathrm{a}}$ \\ ${ }^{a}$ Glenn Department of Civil Engineering, Clemson University, Clemson SC 29634, USA.
}

\begin{abstract}
Highly buoyant plumes, such as wildfire plumes, in low to moderate wind speeds have initial trajectories that are steeper than many industrial waste plumes. They will rise further into the atmosphere before bending significantly. In such cases the plume's trajectory will be influenced by the vertical variation in horizontal velocity of the atmospheric boundary layer. This paper examined the behavior of a plume in an unstratified environment with a power-law ambient velocity profile. Examination of previously published experimental measurements of plume trajectory show that inclusion of the boundary layer velocity profile in the plume model often provides better predictions of the plume trajectory compared to algebraic expressions developed for uniform flow plumes. However, there are many cases in which uniform velocity profile algebraic expressions are as good as boundary layer models. It is shown that it is only important to model the role of the atmospheric boundary layer velocity profile in cases where either the momentum length (square root of source momentum flux divided by the reference wind speed) or buoyancy length (buoyancy flux divided by the reference wind speed cubed) is significantly greater than the plume release height within the boundary layer. This criteria is rarely met with industrial waste plumes, but it is important in modeling wildfire plumes.
\end{abstract}

Keywords: Plume, Bent-Over Plume, Highly Buoyant Plume, Fire Plume, Wildfire, Near Source Behavior

\section{Introduction}

The behavior of a plume in a cross flow, hereafter referred to as a bent-over plume, is ubiquitous in environmental fluid mechanics. Bent-over plumes occur in many situations such as the smoke rising from a chimney (Briggs, 1968; Marro et al., 2014), wastewater discharge into a river (Fischer et al., 2013), and pool fire dispersion (Fisher et al., 2001). Of particular interest in this study are highly buoyant Boussinesq plumes (Morton, 1965). Such have significant generation of vertical momentum and, therefore, have steeper trajectories, and are more influenced by vertical variation in the cross flow velocity. The underlying motivation is to investigate the spread of wildfires through the lofting of firebrands (embers) into the atmosphere by large fire plumes (Sardoy et al., 2007). This study focuses on the role of the fire plume in ember lofting and, therefore, considers neutrally stable atmospheric conditions. The present study does not take into account the effects of convective instabilities such as boundary layer rolls on amplifying the lofting and downwind transport of firebrands (Thurston et al., 2015). Understanding the behavior of large fire plumes blown horizontally by the atmospheric boundary layer is essential to accurate

* Corresponding author

Email addresses: atohidi@g.clemson.edu (Ali Tohidi), nbkaye@clemson.edu (Nigel B. Kaye), Tel .:+1(864)656-5941, Fax:+1 (864)656-2670 (Nigel B. Kaye) 

found that lateral and vertical spreads are unequal, with the lateral spread greater than the vertical spread. Another

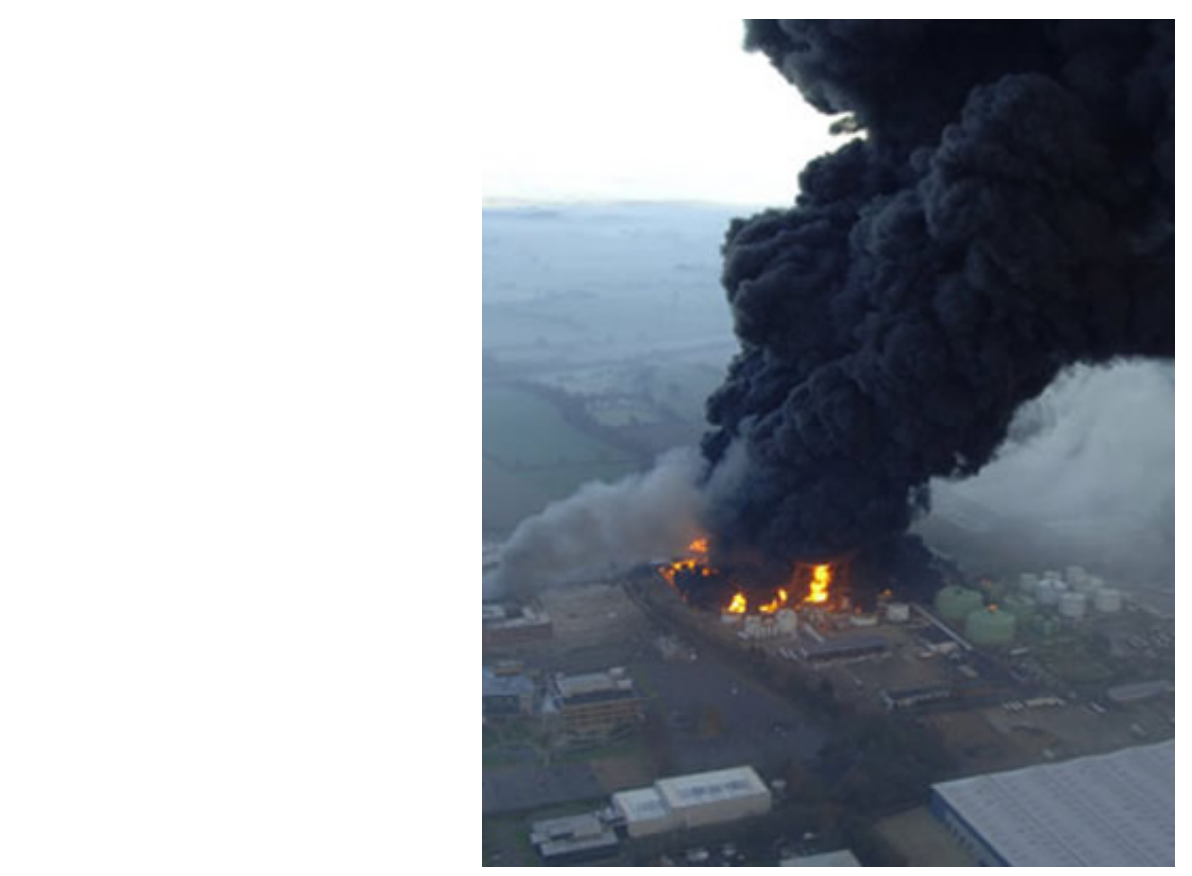

Figure 1: Image of the smoke plume rising from the 2005 Buncefield oil depot fire (copyright Thames Valley Police).

modeling of the lofting and transport of embers which can create spot fires when they land ahead of the main fire front. Bent-over fire plume dynamics are also key to modeling the dispersion of smoke from wildfires.

Simplified models of the rise of a plume into a cross-flow are very well documented in the literature. A detailed analysis of full-scale data as well as laboratory measurements was used by Briggs (1975a,b, 1984) to develop algebraic formulations for plume rise in different environmental conditions. Hoult et al. (1969) extended the local similarity assumption proposed by Morton et al. (1956) to develop a theoretical model for the plume rise in horizontal wind. The model has two empirical parameters to quantify the rate of entrainment of ambient fluid into the plume. Their model assumed that the velocity and density defect profiles are top hat and that the plume cross section is circular. Davidson (1986) discussed the importance of self-similarity profiles of the physical and chemical properties of a plume in these models and concluded that there is no significant difference between employing Gaussian profiles and top-hat profiles. However, the assumption that the plume cross section is circular is less realistic. In many cases, as the plume develops through the cross flow, a counter-rotating vortex pair in the shape of an elongated kidney evolves in the cross-wind direction (Fric \& Roshko, 1994; Smith \& Mungal, 1998). This gives an elliptical form to the plume cross-section, although this vortex pair is not always evident as seen in the Buncefield oil depot fire; See Figure 1. Measurements of the instantaneous plume spread were done by Bennett et al. (1992) and they

assumption made in the model development of Hoult et al. (1969) and Hoult \& Weil (1972) is that the bent-over plume is slender, that is, the plume cross section (radius) is much smaller than the plume center-line curvature's radius. This eliminates the small-scale irregularities due to ambient turbulence (Weil, 1988). Also, Hoult et al. (1969) assumed that the effect of atmospheric turbulence can be ignored because the turbulence intensity within the plume is significantly greater than the ambient turbulence intensity and, as a result, buoyant fluid is not being detrained from the plume envelope. See Hübner (2004) for a more detailed analysis of the conditions under which 
ambient turbulence will break apart a turbulent plume. A brief summary of bent-over plume models is presented in Table 1.

Table 1: Summery of input parameters for various bent-over plume rise models.

\begin{tabular}{|c|c|c|c|c|c|c|c|c|c|c|c|}
\hline & \multicolumn{10}{|c|}{ Models } & \multirow[b]{2}{*}{ 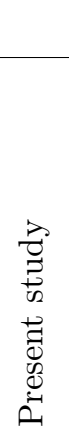 } \\
\hline Assumptions and Properties & 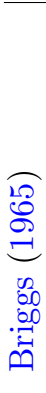 & 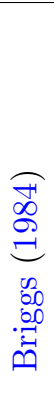 & 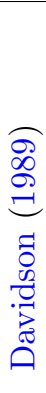 & 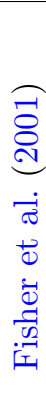 & 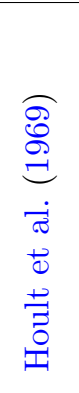 & 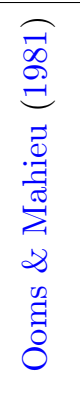 & 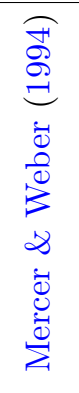 & 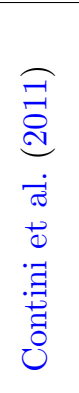 & 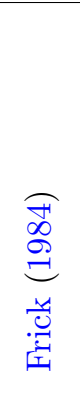 & 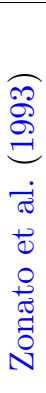 & \\
\hline Restricted to uniform boundary layer & 婃 & 如 & 如 & & & & & & & & \\
\hline No Taylor entrainment & & & & & & & & & 安 & & \\
\hline Circular cross section & 安 & 嫖 & 婃 & 如 & 柿 & 如 & 婹 & - & & & \\
\hline$[\mathrm{T}]$ op hat or $[\mathrm{G}]$ aussian velocity profile & $\mathrm{T}$ & $\mathrm{T}$ & $\mathrm{T}$ & $\mathrm{T}$ & $\mathrm{T}$ & G & $\mathrm{T}$ & $\mathrm{T}$ & G & $\mathrm{T}$ & $\mathrm{T}$ \\
\hline$[\mathrm{P}]$ oint, $[\mathrm{F}]$ inite, or $[\mathrm{L}]$ ine source & $\mathrm{P}$ & $\mathrm{P}$ & $\mathrm{F}$ & $\mathrm{F}$ & $\mathrm{P}$ & $\mathrm{P}$ & $\mathrm{P} / \mathrm{L}$ & $\mathrm{P}$ & $\mathrm{P}$ & $\mathrm{F}$ & $\mathrm{F}$ \\
\hline$[\mathrm{F}]$ orced or $[\mathrm{L}]$ azy plumes & $\mathrm{L}$ & $\mathrm{L}$ & $\mathrm{L}$ & $\mathrm{L}$ & $\mathrm{F} / \mathrm{L}$ & $\mathrm{F} / \mathrm{L}$ & $\mathrm{L}$ & $\mathrm{F} / \mathrm{L}$ & $\mathrm{F} / \mathrm{L}$ & $\mathrm{L}$ & $\mathrm{F} / \mathrm{L}$ \\
\hline$[\mathrm{N}]$ eutral or $[\mathrm{S}]$ tratified environment & $\mathrm{N}$ & $\mathrm{N}$ & $\mathrm{N}$ & $\mathrm{N}$ & $\mathrm{N} / \mathrm{S}$ & $\mathrm{N} / \mathrm{S}$ & $\mathrm{N} / \mathrm{S}$ & $\mathrm{N} / \mathrm{S}$ & $\mathrm{N} / \mathrm{S}$ & $\mathrm{S}$ & $\mathrm{N} / \mathrm{S}$ \\
\hline
\end{tabular}

In the case of wildfire plumes, because they do not, typically, discharge substantial initial momentum, the nearsource flow is dominated by buoyancy-driven acceleration and they are categorized as lazy plumes (see Hunt \& Kaye (2005)). Typically a lazy plume with finite source area cannot be categorized as a slender plume. However, directly above the source the plume contracts until it reaches a minimum radius at a height approximately equal to the source radius. It then begins to expand and approach the self-similar velocity profile. Above this height, the self-similar bent-over plume appears to come from a point source and can be classified as a slender plume (see Baum \& McCaffrey (1989)). Further, estimates of the virtual origin for a highly lazy plume can be obtained using the self-similarity entrainment model of Morton et al. (1956) provided the model is applied above the plume neck (see Kaye \& Hunt (2009)). This indicates that, even if the plume is not technically slender above the neck, the modeling approach of Morton et al. (1956) provides a reasonable description of the flow.

There are a number of differences between wildfires and industrial plumes. For example, wildfires' have an unsteady source buoyancy flux that is controlled by the combustion rate of the fire. While models exist for time varying source flux conditions, see Scase et al. (2006), the time scale over which the fire buoyancy flux varies significantly is large compared to the evolution time of the bent-over plume such that steady models can provide insight into the flow dynamics. Further, wildfires may burn over areas of horizontal extent similar in order to the vertical extent of the boundary layer. Therefore, the results of te present study are mainly applicable to small wildfires and large scale industrial fires such as the Buncefield fire shown in figure 1. For the purpose of this study, the main relevant distinction between wildfire plumes and smaller industrial plumes is that the buoyancy 
flux of a wildfire is orders of magnitude greater than that of industrial plumes. Therefore, wildfire plumes generate significantly more vertical momentum over a greater vertical length scale than typical industrial waste plumes.

As such, wildfire plumes will have steeper trajectories near their source and the influence of vertical variations in time-averaged horizontal ambient velocity in the atmospheric boundary layer will have a greater impact on wildfire plume dynamics.

The goal of the present study is to examine the impact of a power-law boundary layer velocity profile on various bent-over plume parameters and establish the conditions under which the velocity profile needs to be considered in modeling. In Section 2 we review the model of Hoult et al. (1969), present a set of ordinary differential equations for the variation in plume fluxes along the plume centerline trajectory, and discuss alternative entrainment closures. The two entrainment models are compared to previously published experimental measurements of plume trajectory for both uniform and power-law velocity profiles in Section 3. The influence of the plume source conditions on the near field plume trajectory and the conditions under which the boundary layer must be modeled to get accurate trajectory predictions are discussed in Section 4. Full scale examples of when the boundary layer will and will not significantly influence a plume's trajectory are presented in Section 5 and conclusions are drawn in Section 6.

\section{Evolution equations}

In this section the equations of motion for a bent-over plume are presented followed by a discussion of the entrainment formulation and the appropriate choice of entrainment coefficients.

\subsection{Bent-over plume equations}

The evolution equations for the plume's specific volume flux $(Q)$, specific momentum flux $(M)$, specific buoyancy flux $(F)$, and plume centerline trajectory angle $(\theta)$, along the envelope centerline $(s)$ are as follows:

$$
\begin{gathered}
\frac{d Q}{d s}=Q \sqrt{\frac{2\left(1+\lambda^{2}\right)}{M \lambda}}\left(\alpha\left|\frac{M}{Q}-U \cos (\theta)\right|+\beta|U \sin (\theta)|\right) \\
\frac{d M}{d s}-U \cos (\theta) \frac{d Q}{d s}=\frac{F Q}{M} \sin (\theta) \\
U \sin (\theta) \frac{d Q}{d s}+M \frac{d \theta}{d s}=\frac{F Q}{M} \cos (\theta) \\
\frac{d F}{d s}=Q \frac{g}{\varrho_{a}} \frac{d \varrho}{d z} \sin (\theta)=-Q N^{2} \sin (\theta) \\
x=\int_{0}^{s} \cos (\theta) d s \\
z=\int_{0}^{s} \sin (\theta) d s
\end{gathered}
$$

where $U$ is the ambient velocity and $\lambda$ is the ratio of the major to conjugate radii to account for the noncircular cross section of the plume. Ambient stratification is parameterized using the Brunt-Vaisala frequency (N). Equations 
(1-4) express, respectively, conservation of mass, momentum along $s$, transverse momentum, and buoyancy fluxes. A thorough derivation of the equations is presented in Appendix A. For the remainder of the paper we consider only unstratified environments such that $\mathrm{N}=0$, the buoyancy flux $F$ is constant along the plume trajectory, and equation (4) is redundant. The coupled form of equations (1-6) can, therefore, be written in non-dimensional form as:

$$
\left[\begin{array}{ccccc}
1 & 0 & 0 & 0 & 0 \\
-v \cos (\theta) & 1 & 0 & 0 & 0 \\
v \sin (\theta) & 0 & m & 0 & 0 \\
0 & 0 & 0 & 1 & 0 \\
0 & 0 & 0 & 0 & 1
\end{array}\right] \frac{d}{d \eta}\left\{\begin{array}{c}
q \\
m \\
\theta \\
\zeta \\
\xi
\end{array}\right\}=\left\{\begin{array}{c}
\frac{5 q}{6 \alpha \sqrt{m}} I_{s}\left(\alpha\left|\frac{q}{m}-v \cos (\theta)\right|+\beta|v \sin (\theta)|\right) \\
\left(2 q \Gamma_{0} \sin (\theta)\right) /(3 m) \\
\left(2 q \Gamma_{0} \cos (\theta)\right) /(3 m) \\
\cos (\theta) \\
\sin (\theta)
\end{array}\right\}
$$

where $I_{s}=\sqrt{\frac{2\left(1+\lambda^{2}\right)}{\lambda}}$ is a geometric correction term to account for the plume cross-section eccentricity. The other dimensionless parameters are defined as:

$$
q=\frac{Q}{Q_{0}}, \quad m=\frac{M}{M_{0}}, \quad f=\frac{F}{F_{0}}, \quad \xi=\frac{z}{L_{Q_{0}}}, \quad \eta=\frac{s}{L_{Q_{0}}}, \quad \zeta=\frac{x}{L_{Q_{0}}}, \quad v=\frac{U}{u_{0}} .
$$

Here, $u_{0}=M_{0} / Q_{0}$ is the plume source velocity, $\Gamma_{0}=\left(5 Q_{0}{ }^{2} F_{0}\right) /\left(4 \alpha M_{0}{ }^{5 / 2}\right)$, and $L_{Q_{0}}=\left(5 Q_{0}\right) /\left(6 \alpha M_{0}{ }^{1 / 2}\right)$ where the subscript ' 0 ' represents the source value. The parameter $\Gamma_{0}$ is a measure of relative importance of the initial fluxes of buoyancy, momentum, and volume at the source; see Hunt \& Kaye (2005) for a more detailed discussion of source length scales, $\Gamma$, and plume classification. In this study we only apply the model above the plume neck and so the source value of $\Gamma$ is less important and can be thought of as a non-dimensional buoyancy flux.

Equation (7) can be partially decoupled using Gauss-Jordan elimination to give:

$$
\begin{gathered}
\frac{d q}{d \eta}=\frac{5}{6 \alpha} \frac{q}{\sqrt{m}} I_{s}\left(\alpha\left|\frac{q}{m}-v \cos (\theta)\right|+\beta|v \sin (\theta)|\right) \\
\frac{d m}{d \eta}=\frac{2}{3} \Gamma_{0} \frac{q}{m} \sin (\theta)+\frac{5}{6 \alpha} \frac{q}{\sqrt{m}} I_{s}\left(\alpha\left|\frac{q}{m}-v \cos (\theta)\right|+\beta|v \sin (\theta)|\right) v \cos (\theta) \\
m \frac{d \theta}{d \eta}=\frac{2}{3} \Gamma_{0} \frac{q}{m} \cos (\theta)-\frac{5}{6 \alpha} \frac{q}{\sqrt{m}} I_{s}\left(\alpha\left|\frac{q}{m}-v \cos (\theta)\right|+\beta|v \sin (\theta)|\right) v \sin (\theta)
\end{gathered}
$$

For a uniform wind field the far-field plume has a relatively flat trajectory ( $\theta$ is small) and the plume is advected downstream at the ambient wind speed. Therefore, there is no longitudinal entrainment, only transverse entrainment. In this case analysis shows that the fluxes of momentum and volume scale on the trajectory path length raised to the $4 / 3^{\text {rds }}$ power. For a power-law velocity profile the plume is continuously rising into regions of larger ambient velocity. Therefore, even though the trajectory has a small slope, the plume continues to rise into regions of higher velocity and the longitudinal entrainment will remain finite and numerical solution of the equations is required.

\subsection{Entrainment formulation}

The entrainment formulation used in (1) was presented by Hoult \& Weil (1972) and accounts for mixing due to both longitudinal and transverse shear. In this model the entrainment velocity can be written as

$$
u_{e-H}=\alpha\left|\frac{M}{Q}-U \cos (\theta)\right|+\beta|U \sin (\theta)| .
$$


Table 2: Commonly used values of longitudinal $(\alpha)$ and transverse $(\beta)$ entrainment coefficients for top-hat formulations of the bent-over plume equations.

\begin{tabular}{l|c|c}
\hline Closure & $\alpha$ & $\beta$ \\
\hline \hline Hoult \& Weil (1972) & 0.11 & 0.6 \\
Ooms \& Mahieu (1981) & 0.081 & 0.5 \\
Vertical plume (no wind) Lee \& Chu (2003) & 0.12 & - \\
Vertical jet (no wind) Lee \& Chu (2003) & 0.081 & - \\
Cylindrical thermal (no cross wind) Turner (1973) & - & 0.6 \\
\hline
\end{tabular}

This closure is an amalgam of the entrainment models for a vertical jet or plume, see Morton et al. (1956), and that for a horizontal cylindrical thermal. The vertical plume entrainment closure (first term on RHS of (11)) represents entrainment due to longitudinal shear, that is, the difference in velocity between the ambient and the plume in a direction parallel to the local plume trajectory. The cylindrical thermal term (second term on the RHS of (11)) quantifies shear-driven entrainment due to ambient flow normal to the local trajectory. The entrainment mechanism is analogous to that of a long cylindrical thermal rising up into the atmosphere. Entrainment into the thermal scales on the vertical rise height of the thermal which is analogous to the component of the wind speed normal to the plume centerline.

There is an alternate closure formulation presented by Ooms \& Mahieu (1981) based on a work by Abraham (1963). The closure is quite similar to (11) except for a trigonometric correction to the second term that shuts off transverse entrainment when the plume is vertical. The entrainment closure then becomes

$$
u_{e-O}=\alpha\left|\frac{M}{Q}-U \cos (\theta)\right|+\beta|U \sin (\theta)| \cos (\theta) .
$$

This formulation approaches that of $(11)$ in the far field when the trajectory angle approaches zero and $\cos (\theta) \approx 1$. However, in the near field, especially for highly buoyant plumes that have a steep initial trajectory, there is a potentially significant difference. Under the Ooms \& Mahieu (1981) formulation, near the source of a more vertical plume, the transverse entrainment will be significantly reduced and the plume will entrain less horizontal momentum from the ambient flow. Therefore, the Ooms formulation will predict a steeper plume trajectory than (11). This is discussed further in Section 3 where both models are compared to published experimental trajectories.

\subsection{Entrainment coefficient values}

A range of entrainment coefficient values are found in the literature based on either measurements or entrainment in quiescent ambient conditions, or based on fitting model results to plume trajectories measured in the laboratory. Typical values of the entrainment coefficients $\alpha$ and $\beta$ cited in the literature are summarized in table 2. In general, the transverse entrainment coefficient $(\beta)$ is similar to that for a cylindrical thermal Turner (1973). The longitudinal entrainment coefficient $(\alpha)$ used is typically similar to either that for a pure jet or that for a pure plume (Lee \& $\mathrm{Chu}, 2003)$. 
The choice of whether to use the jet or plume entrainment coefficient for the no-wind case is typically based on the source conditions and the vertical extent of the flow. If the source Froude number (based on the source velocity, radius, and reduced gravity) is high then the flow will behave like a jet near the source. For lower values of the Froude number the flow is more like a pure plume. For any finite source of buoyancy the flow will eventually behave like a pure plume (see for example Morton \& Middleton (1973) \& Hunt \& Kaye (2005)). Therefore, there are a number of models for how, under highly forced source conditions, the entrainment coefficient adjusts from that of a jet to the larger plume value (see for example Priestly \& Ball (1955), Fischer et al. (2013) \& Wang \& Law (2002)). There are also a number of explanations for why the plume entrainment coefficient is larger than that of a jet. For example, Kaye (2008) used a simple scaling argument to show that the additional entrainment due to buoyancy generated baroclinic torque matches the behavior of the model of Priestly \& Ball (1955) and the experimental data of Wang \& Law (2002). However, for the case of a bent-over plume, the far field flow is largely horizontal and, therefore, there will be minimal additional buoyancy driven entrainment. Therefore, the longitudinal entrainment will be dominated by longitudinal shear, the jet entrainment coefficient would be more appropriate, and the more sophisticated entrainment models in the literature for vertical plumes would not apply.

The choice of longitudinal entrainment coefficient will effect the modeled plume trajectory. Using the larger plume value of $\alpha$ will result in more near-source entrainment of horizontal momentum and a flatter initial trajectory compared to that predicted using the jet value of $\alpha$. Again, this is of particular importance for flows with initially steep trajectories where longitudinal entrainment is significant over greater distances. In Sect. 3 the entrainment formulations of Hoult \& Weil (1972) and Ooms \& Mahieu (1981) are compared to previously published experimental results. In these plots lines are drawn for each model using both the plume and jet longitudinal entrainment coefficients.

\section{Comparison with previous experiments}

The models presented above are compared to previously published laboratory experiments in order to investigate the influence of the choice of entrainment formulations, entrainment coefficients, and the velocity profile on the trajectory of a bent-over plume. For each experimental test case the data is compared to the bent-over plume equations (1-6) using the entrainment formulation of Hoult (11), the entrainment formulation of Abraham (12), and the algebraic Briggs equation (14) for a plume in a uniform wind field. The test cases are taken from Contini et al. (2011) (cases 1-4), Contini \& Robins (2001) (5-8), Contini et al. (2009) (9-12), and Marro et al. (2014) (1316). The experiments for cases 1-8 were conducted by towing the plume source across a quiescent environment and, therefore, represent plumes in a uniform wind field. The experiments for cases 9-16 were conducted with a finite height plume source in a boundary layer velocity profile.

The velocity profiles in these experiments were well described by a power-law function as proposed by Hellmann (1917) and quantified by Davenport (1960). That is,

$$
U(z)=U_{r}\left(\frac{z}{z_{r}}\right)^{p} ; \quad 0 \leq p \leq 1
$$

where $U_{r}$ is the time averaged horizontal wind speed measured at a reference height $z_{r}$. In general, $p$ varies with wind speed, height range of the fit, upwind topography, and stability of the boundary layer. However, in this 
Table 3: Velocity ratio, source Froude number, velocity profile power-law exponent, and momentum length scale normalized on the release height for the experimental test cases. The test cases are taken from Contini et al. (2011) (cases 1-4), Contini \& Robins (2001) (5-8), Contini et al. (2009) (9-12), and Marro et al. (2014) (13-16).

\begin{tabular}{l|l|c|c|c|c}
\hline Experimental Data & Name & $U_{0} / u_{0}$ & $F r$ & $p$ & $z_{m} / h_{s}$ \\
\hline \hline \multirow{3}{*}{ Contini et al. (2011) } & case (1) & 0.08 & 9.7 & 0 & - \\
& case (2) & 0.15 & 9.7 & 0 & - \\
& case (3) & 0.22 & 9.7 & 0 & - \\
& case (4) & 1.2 & 9.7 & 0 & - \\
\hline \multirow{3}{*}{ Contini \& Robins (2001) } & case (5) & 0.3 & 2.9 & 0 & - \\
& case (6) & 0.52 & 2.9 & 0 & - \\
& case (7) & 0.76 & 2.9 & 0 & - \\
\hline \multirow{5}{*}{ Contini et al. (2009) } & case (8) & 1.2 & 2.9 & 0 & - \\
\hline & case (9) & 0.25 & 84 & 0.23 & 0.042 \\
& case (10) & 0.15 & 126 & 0.23 & 0.063 \\
& case (11) & 0.2 & 70 & 0.23 & 0.059 \\
Marro et al. (2014) & case (12) & 0.13 & 104 & 0.23 & 0.089 \\
\hline & case (13) & 0.76 & 2.9 & 0.21 & 1.287 \\
& case (14) & 0.76 & 4.2 & 0.21 & 0.484 \\
\hline \multirow{5}{*}{ case (15) } & 0.79 & 5.6 & 0.21 & 3.182 \\
& case (16) & 0.74 & 9.6 & 0.21 & 1.524 \\
\hline
\end{tabular}

study, it is only considered to be a function of the upwind terrain surface roughness (see ASCE (1999)). Also, it is worth mentioning that the power-law expression fits best over moderate height range of $20-300$ meters through the boundary layer (Cook, 1997) as opposed to the logarithmic fit that does not show a good representation of the boundary layer velocity above the inertial sub-layer heights of approximately 200 meters, see Tieleman (2008) \& Li et al. (2010). The experimental conditions for each test case are summarized in Table 3 in terms of the source Froude number $\left(F r=u_{0} / \sqrt{g^{\prime} D}\right)$, relative wind speed $\left(\mu=U_{0} / u_{0}\right)$, the boundary layer power-law exponent $(p)$, and the non-dimensional release height $\left(h_{s} / D\right)$. Where $U_{0}$ is the effective horizontal velocity of the boundary layer at the release height of the plume, and $u_{0}$ is the plume exit velocity.

( The results of the comparison for the uniform velocity profile experiments (cases $1-8)$ are presented in Figures 2
$\& 3$. For each plot in Figure $2 \& 3$ the lengths of the vertical axis and horizontal axis are the same to allow
for direct trajectory slope comparison between plots. The first four cases, in Figure 2 , have relatively low wind
speeds $(\mu \leq 0.22$ ) except for case 4 where $\mu=1.2$. However, they are quite highly forced $(F r=9.7)$ and so there is
relatively little buoyancy-generated vertical momentum downstream of the plume source. Cases 5 - 8 , in Figure 3 , are
more buoyant $(F r=2.9)$ and have higher wind speeds, mostly $U_{0} / u_{0} \geq 0.3$. The impact of the additional buoyancy
can be seen by comparing the trajectory for case 5 with that of case 3 . Case 5 has the higher relative wind speed
$(\mu=0.3$ compared to 0.22 for case 3 ) but also has a steeper trajectory due to the additional buoyancy-generated 
vertical momentum.

For all eight cases the integral model performs quite well when using the Hoult entrainment formulation (11). For this formulation there is little difference in the trajectories based on the choice of longitudinal entrainment coefficient $(\alpha)$. The Abraham formulation (12) performs less well, overpredicting the plume height for all cases. This is due to the reduction in entrainment of horizontal momentum resulting from the $\cos (\theta)$ modification to the transverse entrainment term. These steeper trajectories also exhibit greater variation in model results with the choice of $\alpha$ as longitudinal entrainment of horizontal momentum plays a greater role near the source due to transverse entrainment being shut off. When the lower jet value of $\alpha$ is used the plume has a steeper trajectory due to lower entrianment of horizontal momentum. The Briggs generalized algebraic equation (Briggs, 1984; Davidson, 1989) which relates the plume rise height to its downwind distance as

$$
z(x)=\left[\frac{3 M x}{\beta_{1}^{2} U_{0}^{2}}+\frac{3 F x^{2}}{2 \beta_{2}^{2} U_{0}^{3}}\right]^{1 / 3}
$$

where

$$
\beta_{1}=0.4+1.2 \frac{U}{u_{0}} \quad, \quad \beta_{2}=0.6,
$$

provides the best agreement across all the uniform-velocity profile cases, that is cases $1-8$. This is primarily because the Briggs equation is an empirical curve fit based on a theoretical functional form and is, therefore, tuned to match experimental results. Whereas, the entrainment models in Hoult and Abraham were integrated using constant entrainment coefficients (given in Table 2) based on experiments run in quiescent environments. Therefore, the entrainment models capture more of the flow physics but will perform less well for cases similar to those from which the Briggs equation was developed.

The comparison between the models and experiments conducted using a boundary layer velocity profile are shown in Figures 4 (cases 9-12) and 5 (cases 13-16). In both figures the plume trajectory is plotted relative to the plume source height. The axis length ratios are the same as for Figure $2 \& 3$ so direct visual comparison of trajectory slope can be made. In all cases the plume equations are solved using the boundary layer power-law velocity profile fit specified in the original papers. Cases 9-12 are much more forced $(F r \geq 70)$ than cases 13-16 $(F r \leq 9.6)$. Cases 9-12 were also run with a much lower relative wind speed $\left(U_{0} / u_{0} \leq 0.25\right)$ than cases $13-16\left(U_{0} / u_{0} \geq 0.74\right)$. In all eight cases the boundary layer power law exponents are very similar, see Table 3.

For cases 9-12 the algebraic Briggs equation (14) provides the best agreement with the experimental data even though the experiments were run for a non-uniform velocity profile. However, for cases 13-16, the Hoult formulation (11) is clearly the most accurate. The only significant difference between the two sets of tests is the release height of the plume above the ground. The release heights scaled on the source diameter for cases 9-12 range from 43 to 60 whereas for cases $13-16$ the $h_{s} / D=1.5$. Therefore, the plumes from cases 13-16 will experience a greater increase in horizontal velocity variation with height than the plumes from cases 9-12 due to higher wind shear near the boundary, and, therefore, the role of the boundary layer in flattening out the trajectory is greater.

The comparison of various bent-over plume models with laboratory measurements of plume trajectory in a boundary layer indicate that there are circumstances where the algebraic Briggs equation provides the best trajectory prediction (cases 9-12). However, there are also cases where the full set of plume equations must be solved, with 

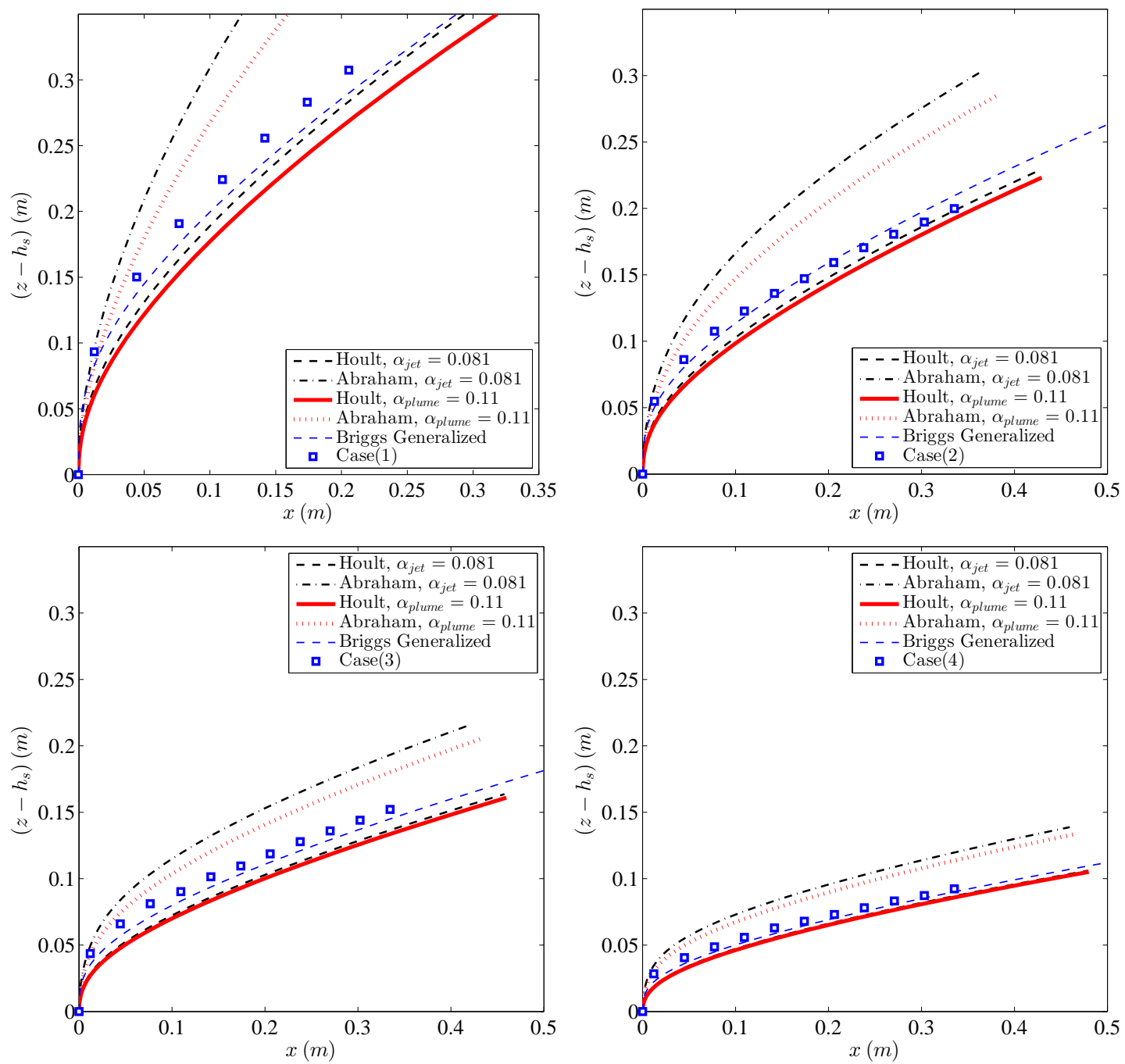

Figure 2: Plots of experimental trajectory (squares) for cases 1-4 (left to right and down) listed in Table 3 along with model results for Hoult \& Weil (1972) - solid \& thick dashed lines, Abraham (1963)/Ooms \& Mahieu (1981) - dot-dashed \& dotted lines, and the Briggs (1984) generalized algebraic equation (14) - thin dashed line.

the boundary layer velocity profile included, in order to get an accurate trajectory prediction (cases 13-16). The conditions under which the full solution of the plume equations is required is discussed next.

\section{Influence of source conditions}

For a plume in a wind field with a power-law velocity profile the far-field plume fluxes of volume and momentum will be significantly greater than the source fluxes and the plume dynamics will only depend on the wind conditions and the plume buoyancy flux. However, this may be so far downwind as to not be of any practical significance. It is, therefore, important to understand the conditions under which the boundary layer flow significantly influences the near source flow. In this section we present a dimensional analysis of the near source flow conditions and derive a set of conditions under which the boundary layer must be modeled to correctly capture the near field flow trajectory. 

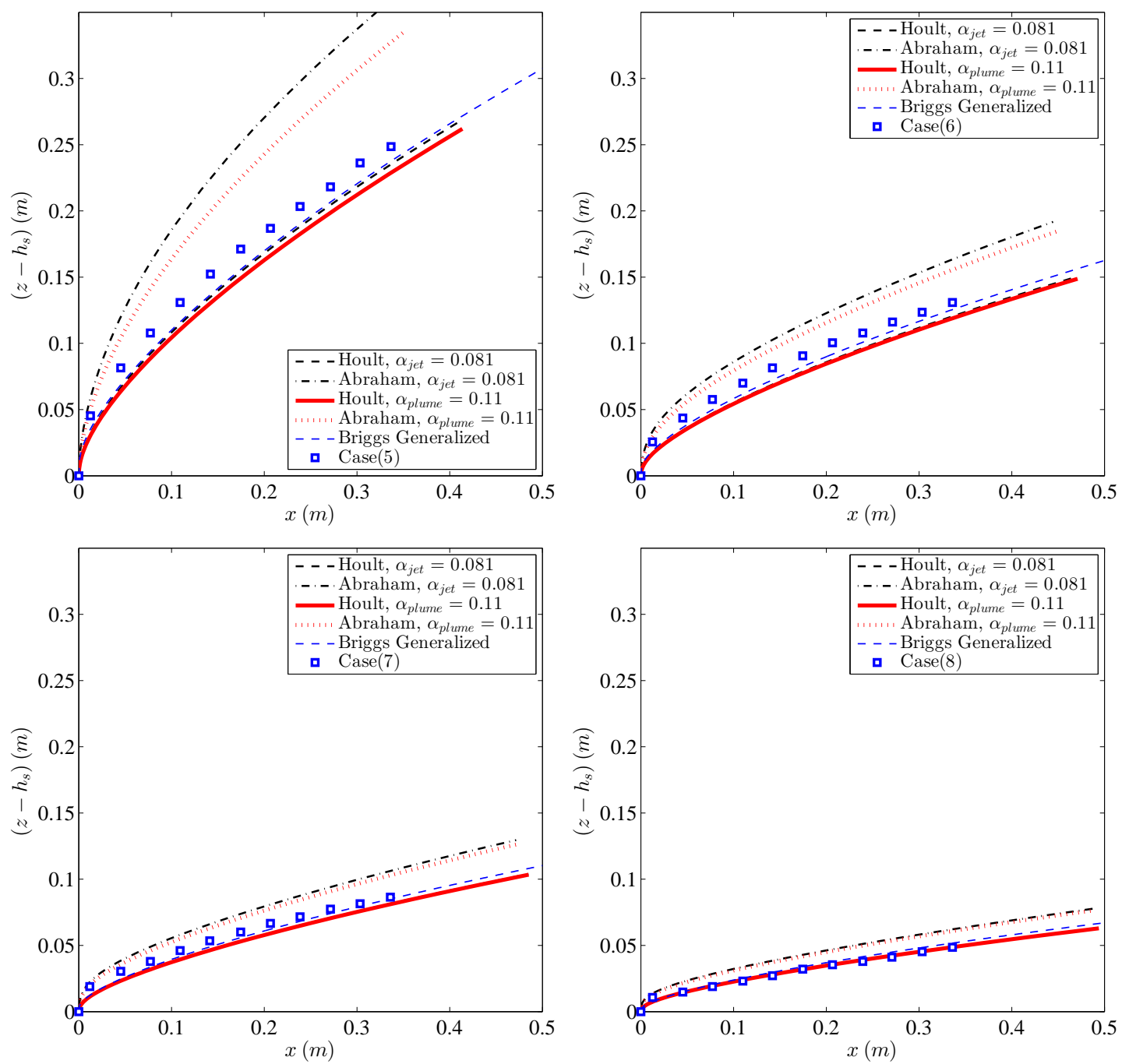

Figure 3: Plots of experimental trajectory (squares) for cases 5-8 (left to right and down) listed in Table 3 along with model results for Hoult \& Weil (1972) - solid \& thick dashed lines, Abraham (1963)/Ooms \& Mahieu (1981) - dot-dashed \& dotted lines, and the Briggs (1984) generalized algebraic equation (14) - thin dashed line.

\subsection{Uniform velocity profile $(p=0)$}

For a uniform velocity profile the plume source conditions can be written in terms of the source diameter $(D)$, source reduced gravity $\left(g^{\prime}\right)$, and the exit velocity $\left(u_{0}\right)$. The wind conditions can be parameterized by the mean horizontal wind speed $\left(U_{0}\right)$. This leads to two non-dimensional groups, the source Froude number and exit velocity ratio (referred to herein as the relative wind speed) given by

$$
F r=\frac{u_{0}}{\sqrt{g^{\prime} D}} \quad \text { and } \quad \mu=\frac{U_{0}}{u_{0}}
$$

respectively; See Lee \& Chu (2003). All non-dimensional downstream parameters will be functions only of these two parameters, distance from the source, the choice of entrainment closure, and the choice of entrainment coefficients. Distance from the source can be characterized in terms of the distance along the plume centerline, downwind distance, or vertical distance. As we are primarily concerned with the influence of ABL flows on bent-over plumes, we focus on the vertical distance $(z)$. 

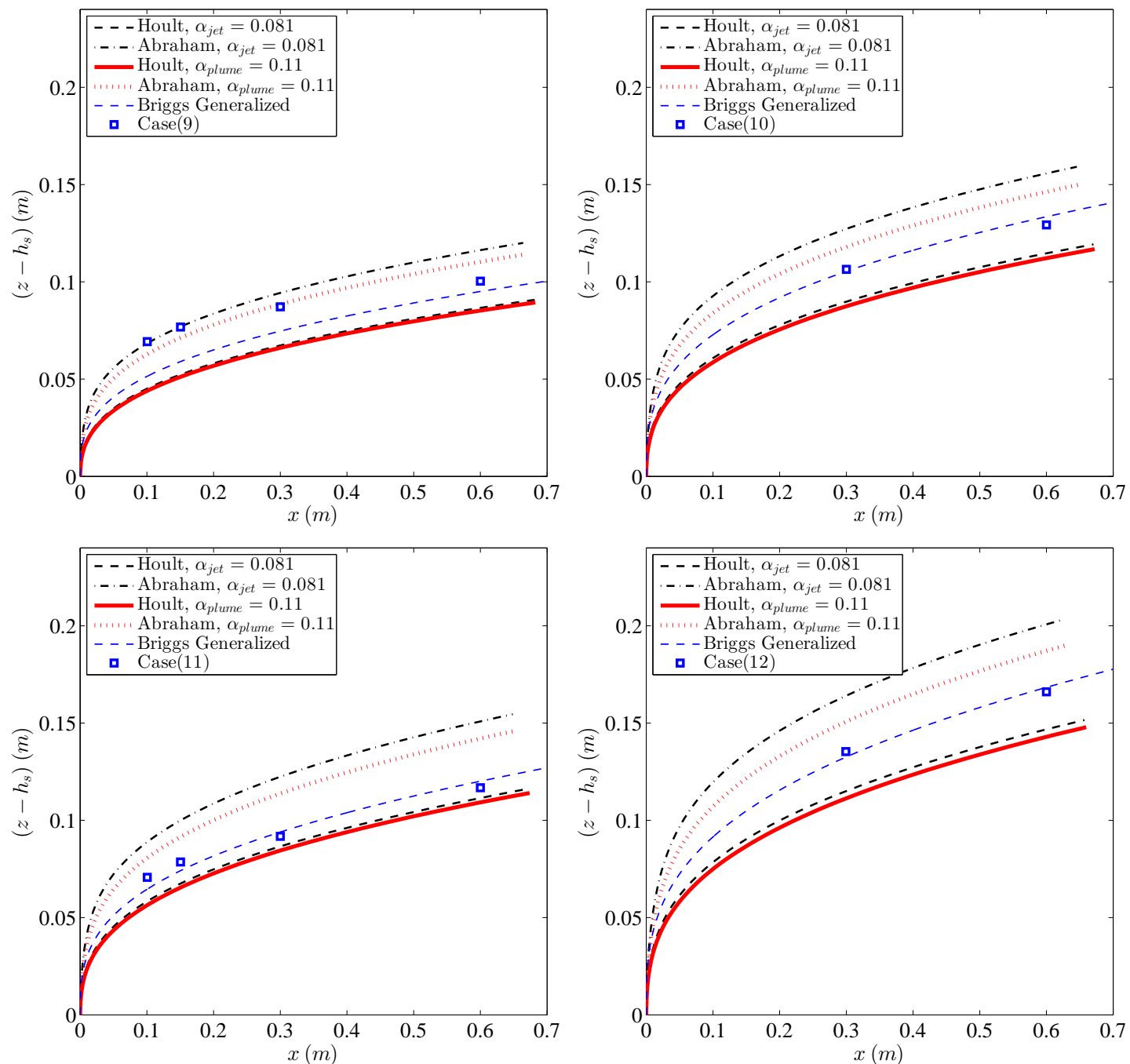

Figure 4: Plots of experimental trajectory (squares) for cases 9-12 (left to right and down) listed in Table 3 along with the model results. Lines are the same as for Figure 2.

This height can be non-dimensionalized in terms of any number of length scales but the most commonly used length scales are the momentum length, buoyancy flux length, and source diameter. The first two are given by

$$
z_{m}=\frac{M_{0}^{1 / 2}}{U_{0}}=\frac{u_{0} D}{U_{0}}=\frac{D}{\mu} \quad \text { and } \quad z_{f}=\frac{F_{0}}{u_{0}^{3}}=\frac{u_{0} D^{2} g^{\prime}}{U_{0}^{3}}=\frac{D}{\mu^{3} F r^{2}}=\frac{z_{m}}{\mu^{2} F r^{2}}
$$

respectively. The momentum length $\left(z_{m}\right)$ represents the vertical distance at which a vertical pure point source jet will have a vertical mean velocity equal to the mean wind speed. It, therefore, represents a vertical scale over which the bent-over jet will have a relatively steep trajectory. The buoyancy length $\left(z_{f}\right)$ represents the vertical distance at which a vertical pure point source plume will have a mean vertical velocity equal to that of the mean wind speed. It therefore represents a second vertical scale over which the bent-over plume will have a relatively steep trajectory. Which of these two vertical scales is most appropriate depends on the balance of momentum and buoyancy fluxes at the source $(F r)$ and the relative wind speed $(\mu)$. To illustrate this, the height at which the plume has a trajectory angle of $45^{\circ}$ (denoted by $z_{45}$ ) was calculated for a broad range of $F r$ and $\mu$ by integrating the plume equations (1-6) along the centerline trajectory $(s)$ using MATLAB's built-in ODE solver (ode15s). Contour plots of $z_{45}$ scaled on 

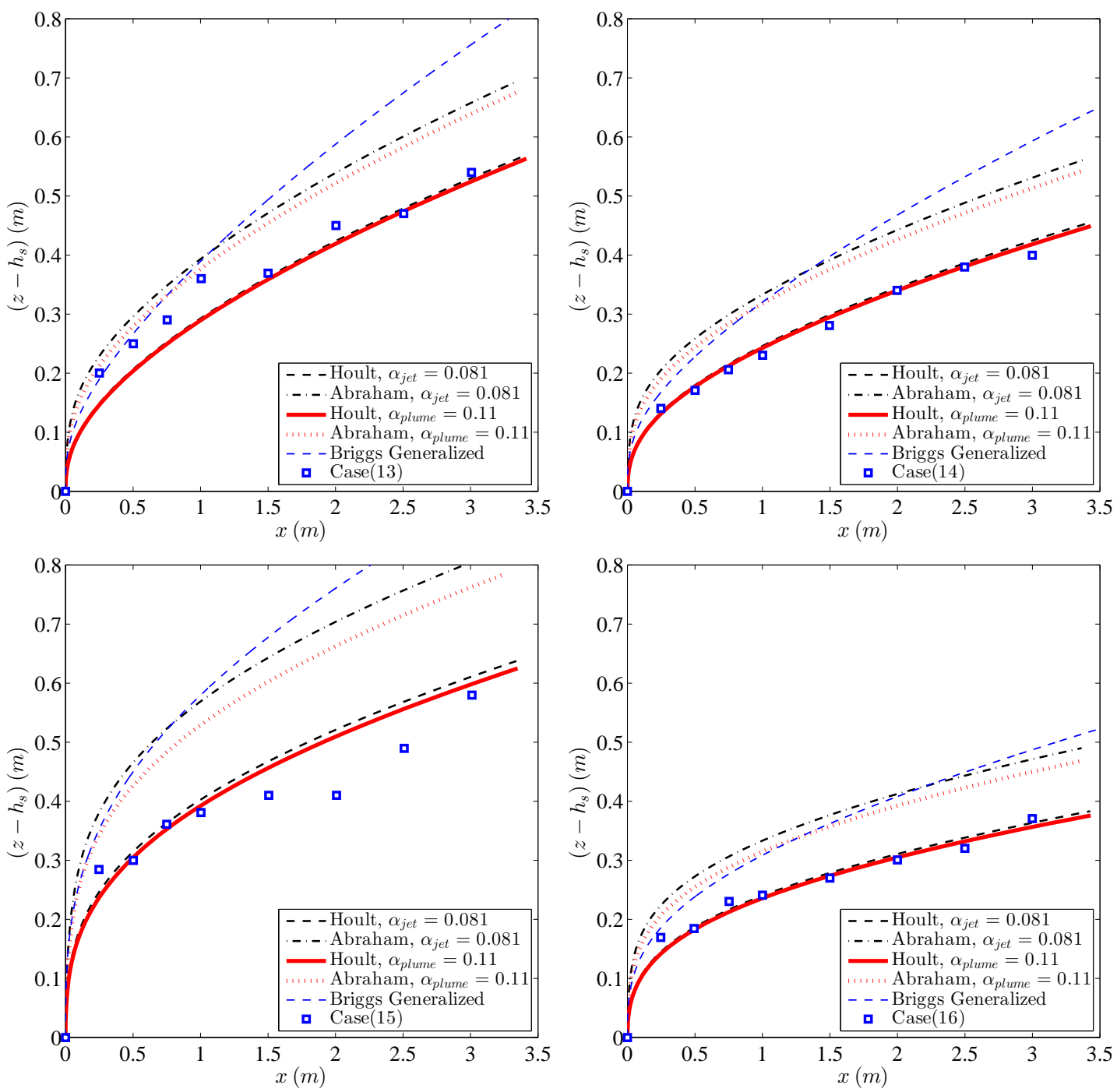

Figure 5: Plots of experimental trajectory (squares) for cases 13-16 (left to right and down) listed in Table 3 along with the model results. Lines are the same as for Figure 2.

the two length scales $z_{m}$ and $z_{f}$ are shown in Figure 6.

Figure 6 (left) shows $\Pi_{45 . m}=z_{45} / z_{m}$ in $(F r, \mu)$ space. For much of the parameter space $\Pi_{45 . m}$ is independent of the Froude number (horizontal contours) indicating that, in these regions, $z_{m}$ is the appropriate vertical scale for characterizing the rate of bending of the plume. The horizontal contours are seen for large relative wind speed regardless of Froude number and large Froude numbers regardless of the relative wind speed. For large source Froude numbers the source momentum dominates and the flow behaves as a jet. As such the near field bending of the flow is controlled by the momentum length $z_{m}$. However, for small Froude numbers and large relative wind speed $(\mu)$ the bending also scales on $z_{m}$ even though the plume source is buoyancy dominated. This is due to the length scales being defined in terms of pure point sources of either momentum or buoyancy. For higher wind speeds the plume will bend over more rapidly and $z_{45}$ will get very small. In this limit $\left(z_{45} \rightarrow 0\right)$ the velocity of the point source of momentum (a pure jet) $\left(u_{j e t} \sim z^{-1}\right)$ is larger than the velocity of the point source of buoyancy (a pure plume) $\left(u_{\text {plume }} \sim z^{-1 / 3}\right)$. Therefore, even though the source is buoyancy-dominated, for high wind conditions the 

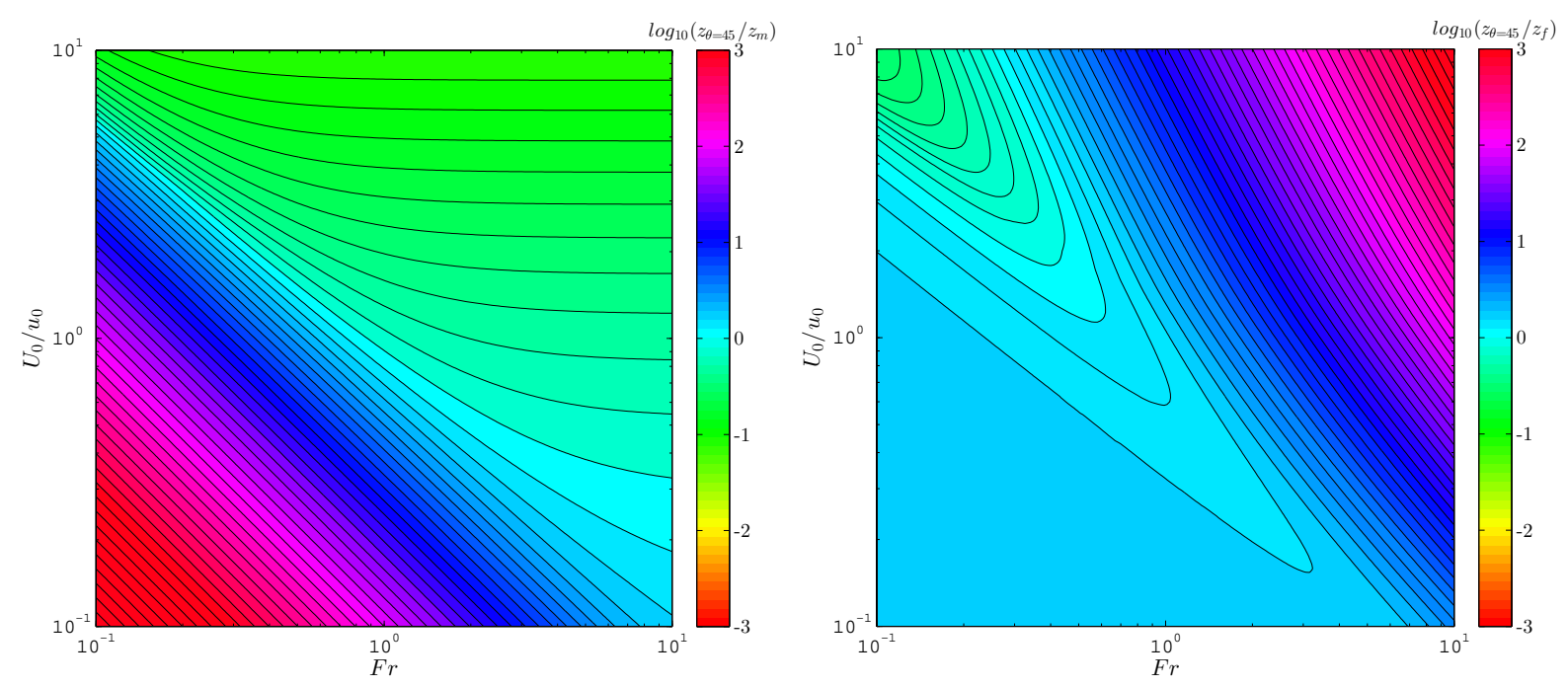

Figure 6: Log-log scale contour plots of $z_{45}$ as a function of $F r$ and $\mu$ with $z_{45}$ scaled on (left) $z_{m}$ and (right) $z_{f}$.

buoyancy does not have adequate vertical distance to generate enough vertical momentum to influence the height at which it becomes significantly bent over. Another way of establishing this result is to consider the relative values of the two length scales. The height at which the plume is significantly bent over will be controlled by the larger of the two source length scales. In a uniform boundary layer one can write the ratio of the length scales as

$$
\frac{z_{m}}{z_{f}}=\mu^{2} F r^{2}
$$

indicating that the appropriate length scale will be the momentum length provided $\mu \mathrm{Fr} \gg 1$.

Figure 6 (right) shows $\Pi_{45 . f}=z_{45} / z_{f}$ in $(F r, \mu)$ space. In this case $\Pi_{45 . f}$ is constant for $\mu F r \ll 1$. In fact, in this region the bending height is independent of both $\mu$ and $F r$, whereas when $z_{m}$ was the controlling length scale, the bending height was still a function of $\mu$. This is because, for small Froude numbers, the plume velocity is controlled by the buoyancy flux and is, therefore, independent of the plume source velocity and, hence, independent of $\mu$.

\subsection{Power-law velocity profile $(p>0)$}

The introduction of a power-law ambient velocity profile adds two new parameters to the problem: the power law exponent $p$ and the plume release height $h_{s}$. There are, therefore, two additional non-dimensional groups that, without loss of generality, can be written as

$$
p \quad \text { and } \quad \psi=\frac{z_{m}}{h_{s}} \text {. }
$$

For the boundary layer velocity profile the reference velocity is taken to be the velocity at the plume release height and the other non-dimensional groups are unchanged. For any boundary layer cross flow the plume will experience higher wind speeds than the reference wind speed at all heights above the source and will have a flatter (less steep) trajectory. For larger power-law exponents $(p)$ the rate of increase in wind speed with height is greater and the plume will bend over more rapidly. Conversely, the higher the release height (the smaller $\psi$ ) the lower the level of 

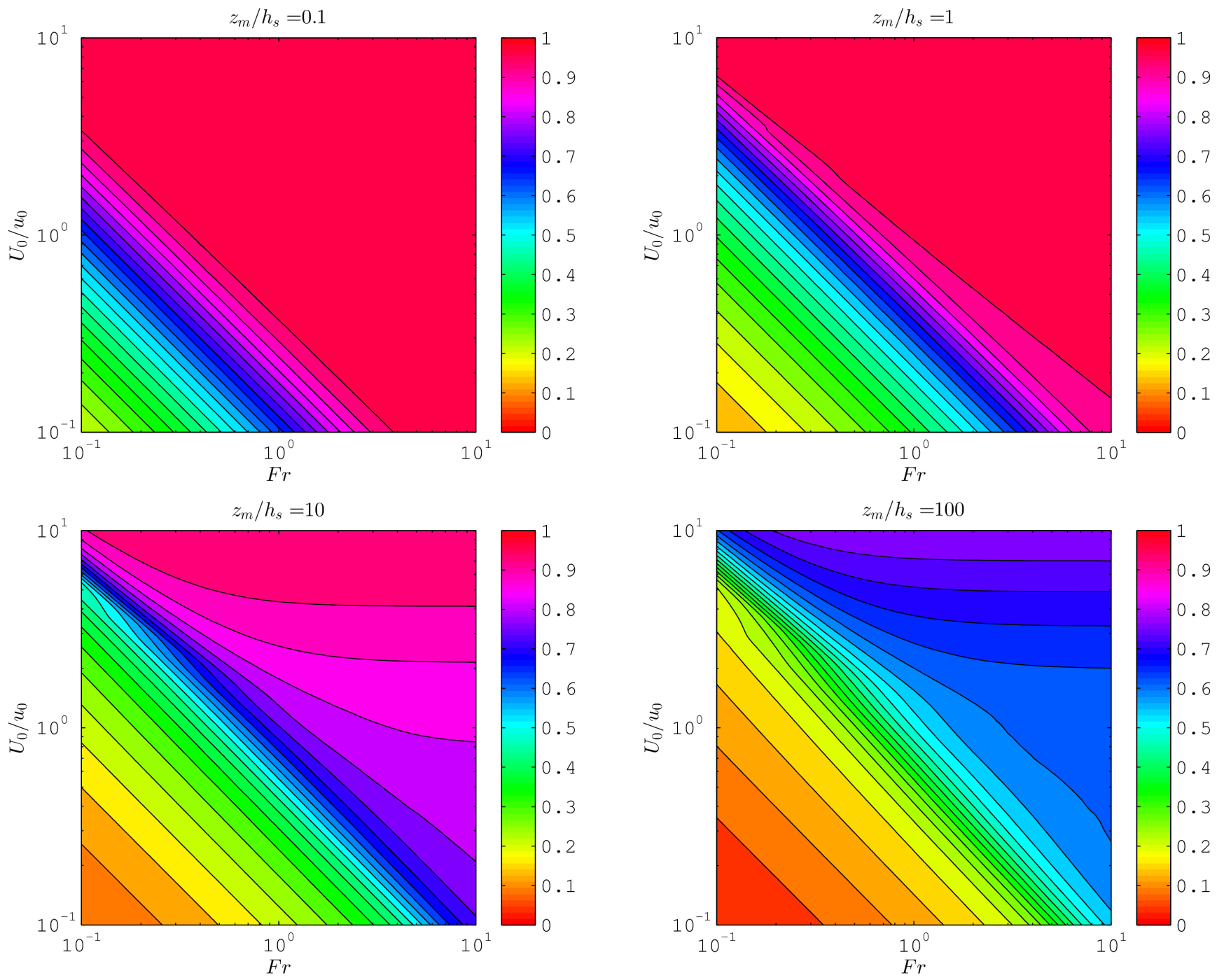

local wind shear experienced by the plume and the steeper the trajectory compared to a lower release height into the same boundary layer.

This behavior is summarized in Figures 7-10 which show contour plots of $z_{45}$ in the power-law boundary layer, i.e. $z_{45 . p}$, normalized on $z_{45}$ in the uniform velocity profile for the same $F r$ and $\mu$. This is denoted by $\chi=z_{45 . p} / z_{45 . u}$. The contour plots show that in all cases the height at which the plume path is at $45^{\circ}$ is lower for the boundary layer flow than for the equivalent uniform velocity profile case. As expected, for small $p$ and $z_{m} / h_{s}$ the trajectory is hardly changed (Figures $7-10(\mathrm{a}, \mathrm{b}))$. However, for lower release height (larger $z_{m} / h_{s}$ ) there is a significant flattening of the trajectory due to the boundary layer velocity profile. Further, in all cases the trajectory is flatter for low Froude number plumes in a relatively low velocity wind field. In these cases the vertical momentum is buoyancy- rather than source-generated, the wind speed is low, and the plume has a steeper initial trajectory. Therefore, it reaches higher into the boundary layer where the wind speed is greater and is bent over more rapidly compared to the same plume in a uniform wind field.
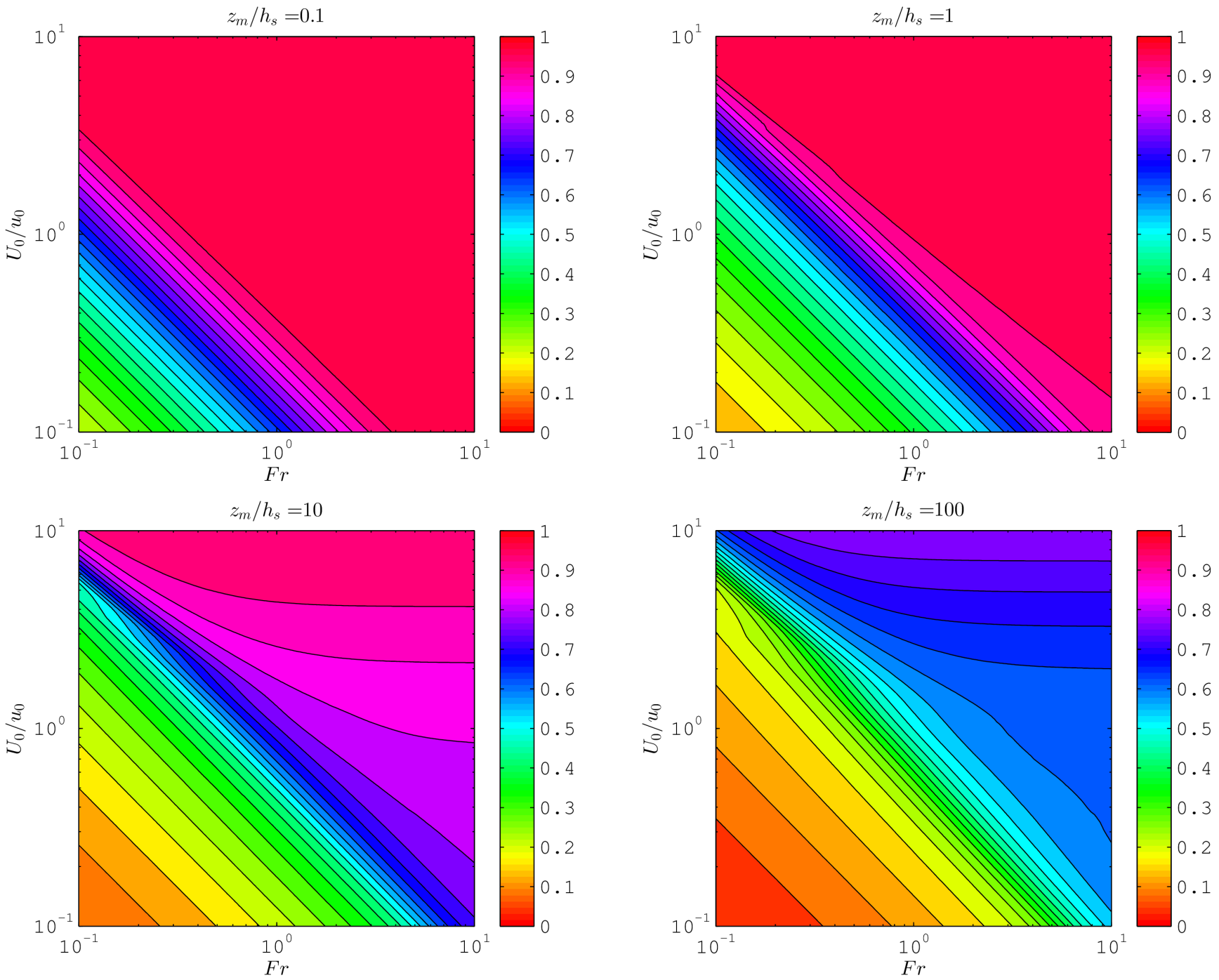

Figure 7: Contour plots of $\chi=z_{45 . p} / z_{45 . u}$ as a function of $F r$ and $\mu$ for $p=0.1$ and (a) $z_{m} / h_{s}=0.1 ;(\mathrm{b}) z_{m} / h_{s}=1 ;(\mathrm{c})$ $z_{m} / h_{s}=10 ;(\mathrm{d}) z_{m} / h_{s}=100$. 

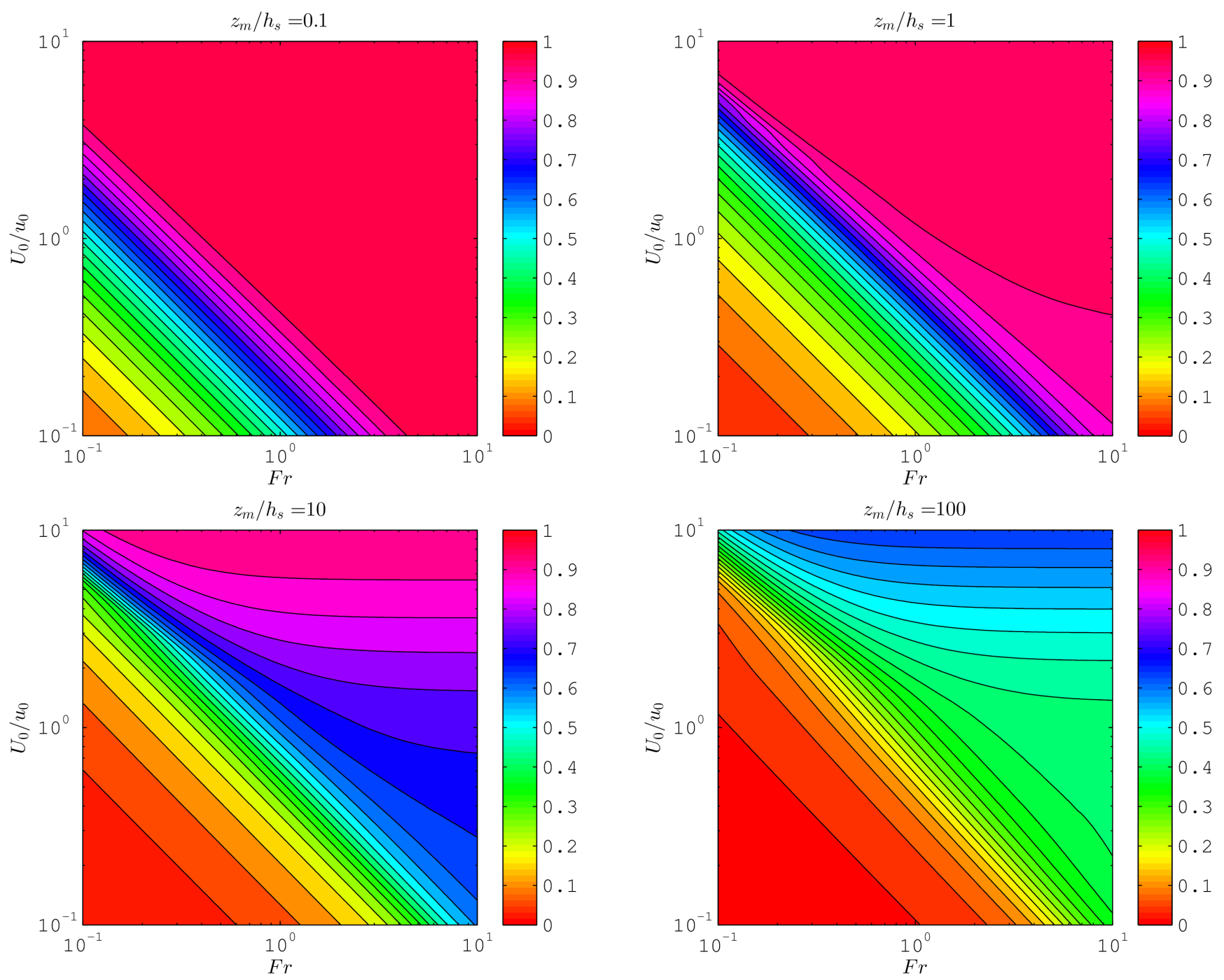

Figure 8: Contour plots of $\chi=z_{45 . p} / z_{45 . u}$ as a function of $F r$ and $\mu$ for $p=0.2$ and (a) $z_{m} / h_{s}=0.1 ;(\mathrm{b}) z_{m} / h_{s}=1 ;(\mathrm{c})$ $z_{m} / h_{s}=10 ;(\mathrm{d}) z_{m} / h_{s}=100$.

An alternative way of viewing this is to consider the definitions of the vertical length scales $z_{m}$ and $z_{f}$. For a power-law velocity profile the length scale can be modified to account for the vertical variation in $U$. Equating the pure plume velocity scaling

$$
u \sim F^{1 / 3}\left(z-h_{s}\right)^{-1 / 3}
$$

with the power-law velocity profile (13) in which the vertical coordinate is measured from the plume release height leads to

$$
z_{f}\left(\frac{z_{f}+h_{s}}{h_{s}}\right)^{3 p}=\left(\frac{F_{0}^{1 / 3}}{U_{0}}\right)^{3} \quad \text { at } \quad z=z_{f}+h_{s}
$$

which can be solved implicitly for $z_{f}$. The equivalent definition for $z_{m}$ is

$$
z_{m}\left(\frac{z_{m}+h_{s}}{h_{s}}\right)^{p}=\frac{M_{0}^{1 / 2}}{U_{0}} \quad \text { at } \quad z=z_{m}+h_{s} .
$$

When $p=0$ both definitions reduce to the uniform velocity profile definitions given in (17). 

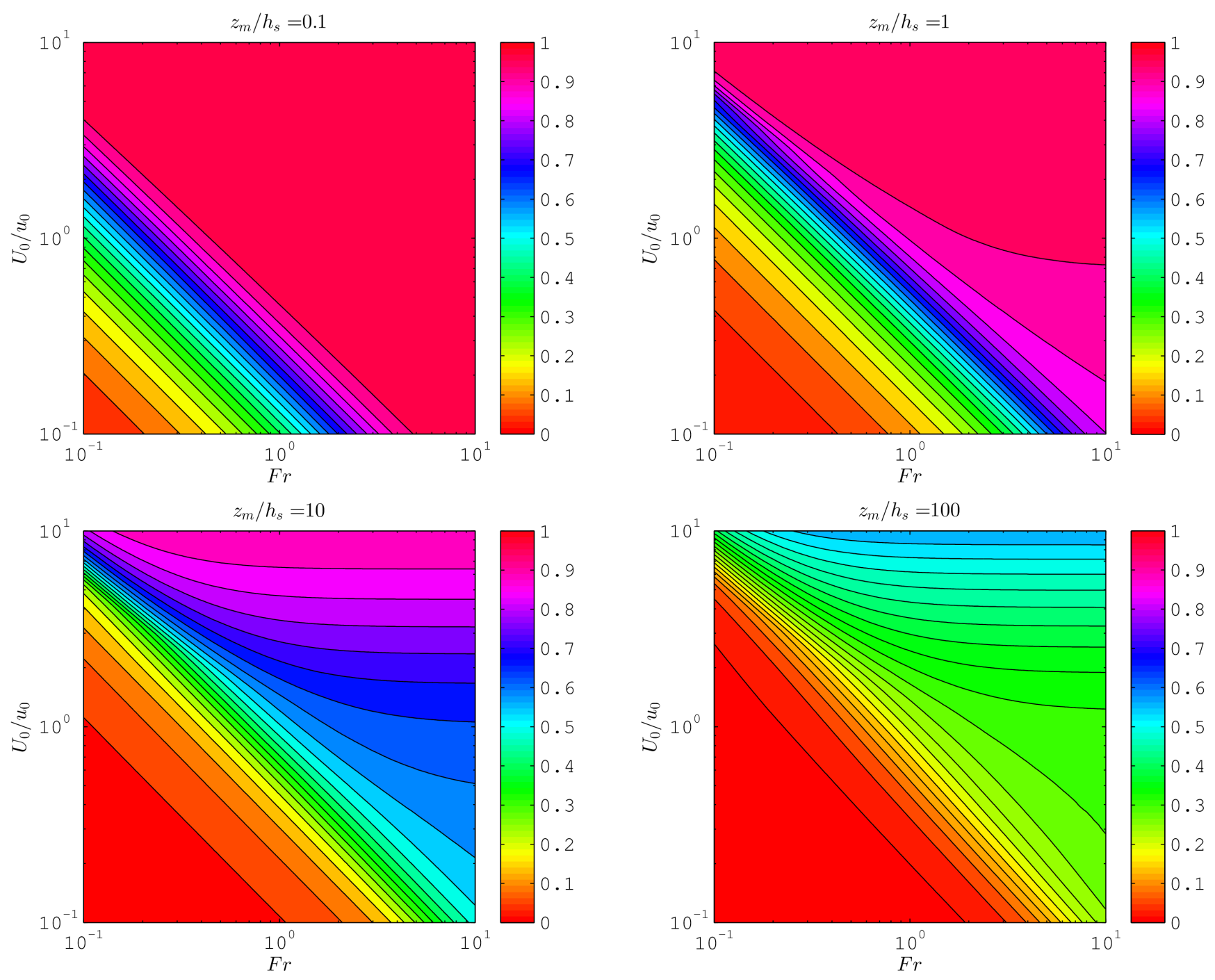

Figure 9: Contour plots of $\chi=z_{45 . p} / z_{45 . u}$ as a function of $F r$ and $\mu$ for $p=0.3$ and (a) $z_{m} / h_{s}=0.1$; (b) $z_{m} / h_{s}=1$; (c) $z_{m} / h_{s}=10 ;(\mathrm{d}) z_{m} / h_{s}=100$.

In both (21) and (22) the righthand side of the equation is identical to the righthand side of the definitions for a uniform velocity profile (17) and the term in brackets on the lefthand side is greater than 1 . Therefore, for $p>0$ and $h_{s}>0$ the values of $z_{f}$ and $z_{m}$ will be smaller than their uniform velocity profile definitions. The greater the difference between the uniform and boundary layer definitions of $z_{m}$ and $z_{f}$, the greater the influence of the boundary layer on the plume trajectory. This will be the case when

$$
\varsigma_{f}=\frac{z_{f \cdot u}}{z_{f \cdot p}}=\left(\frac{z_{f}}{h_{s}}+1\right)^{3 p} \gg 1 \quad \text { or } \quad \varsigma_{m}=\frac{z_{m . u}}{z_{m \cdot p}}=\left(\frac{z_{m}}{h_{s}}+1\right)^{p} \gg 1
$$

where the subscripts ' $u$ ' and ' $p$ ' refer to the uniform and power-law velocity profiles respectively. This is consistent with the contour plots in Figures 7-10 which show significant flattening of the plume trajectory for larger values of $z_{m} / h_{s}, p$, and $z_{f} / h_{s}=\left(z_{m} / h_{s}\right) /\left(\mu^{2} F r^{2}\right)$. It is also consistent with the observation in the previous section that experimental cases 13-16 were more strongly influenced by the boundary layer than cases 9-12. Substituting experimental values into (23) gives $1.1<\varsigma_{f}<1.3$ and $2.4<\varsigma_{m}<2.8$ for cases 9-12 compared to $8.3<\varsigma_{f}<33$ and 

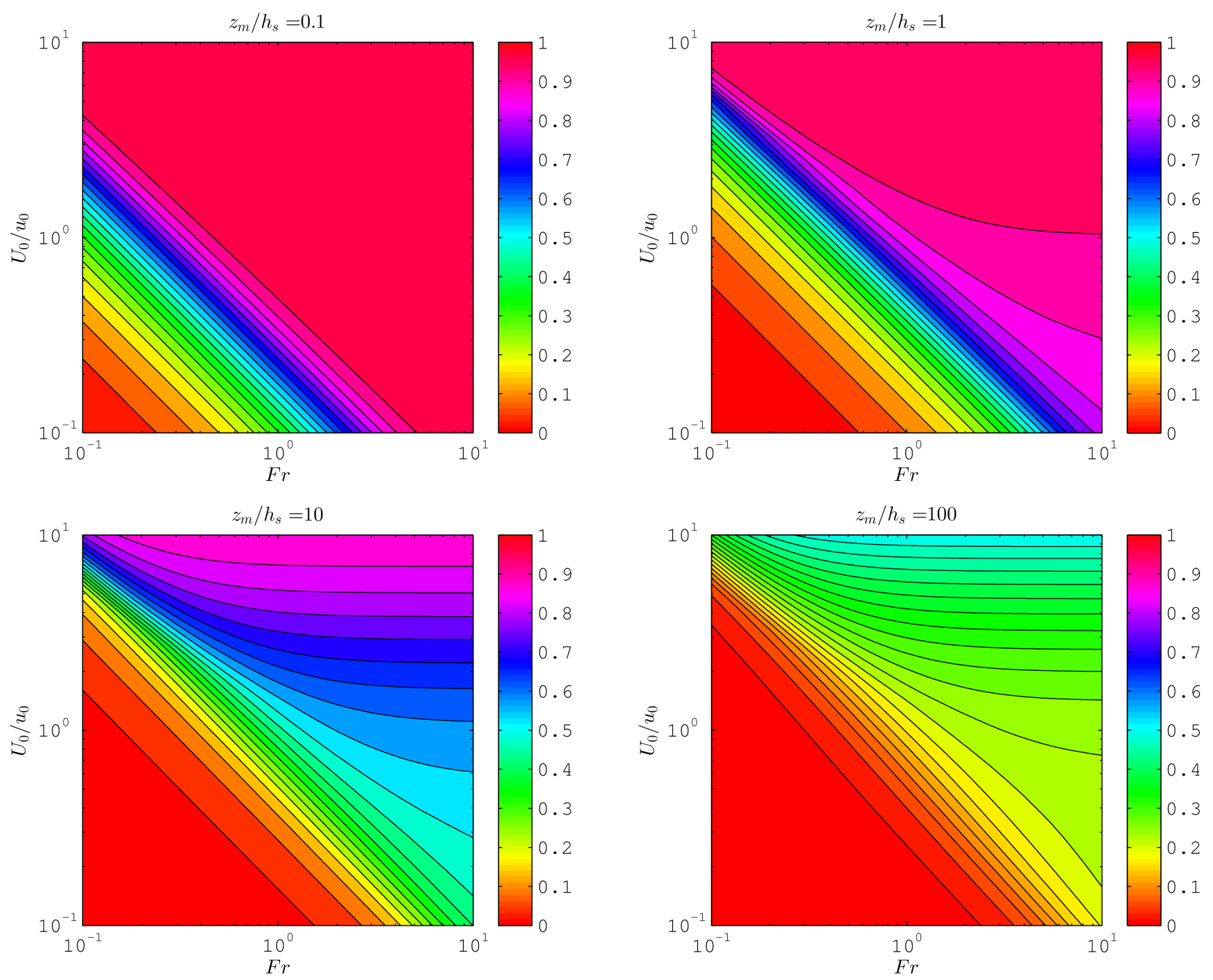

Figure 10: Contour plots of $\chi=z_{45 . p} / z_{45 . u}$ as a function of $F r$ and $\mu$ for $p=0.4$ and (a) $z_{m} / h_{s}=0.1$; (b) $z_{m} / h_{s}=1$; (c) $z_{m} / h_{s}=10 ;$ (d) $z_{m} / h_{s}=100$.

$4.4<\varsigma_{m}<5.4$ for cases 13-16. Examples of when this is significant in full-scale applications are discussed in the next section.

\section{Model selection criteria case studies}

The previous section established a criteria for when the vertical velocity variation in the atmospheric boundary layer will significantly effect a plume's trajectory (Equation 23) such that the algebraic Briggs equation will no longer give an accurate trajectory prediction. This section illustrates the use of this criteria by applying it to case studies of full-scale industrial and wildfire plumes. The case study parameters are presented in Table 4 and represent six different smoke stacks and two examples of wildfires. In general the smoke stacks produce forced plumes $(F r>1.2)$. Wildfires are highly lazy large area plumes that accelerate and contract above the fire. However, as the entrainment equations have been shown to be inappropriate in the near source region below the plume neck (Linden \& Kaye, 2006) the plumes are modeled as pure plumes above the neck and the flow below the neck is not modeled. 
Table 4: Ranges of stack and atmospheric boundary layer parameters for the plume cases (1-6) are excerpted from Carson \& Moses (1969). Parameter values in case (7) are adopted based on Bhutia et al. (2010) for small-scale wildfires.

\begin{tabular}{l|l|c|c|c|c|c|c|c}
\hline Case & Case Name & $U_{0}(\mathrm{~m} / \mathrm{s})$ & $u_{0}(\mathrm{~m} / \mathrm{s})$ & $P_{o}(M W)$ & $h_{s}(\mathrm{~m})$ & $D(\mathrm{~m})$ & $F r$ & Symbol \\
\hline \hline 1 & Harwell & $11.7-3.0$ & 9.9 & 5.1 & 61 & 3.46 & 4.4 & $\times$ \\
2 & Duisburg & $10.5-1.6$ & $12.1-3.5$ & $10.4-4.1$ & 125 & 3.5 & $1.0-4.2$ & $\square$ \\
3 & Gernsheim & $9.2-1.5$ & $5.3-2.2$ & $4.2-1.5$ & 75 & 2.3 & $0.7-1.5$ & $o$ \\
4 & TVA-Widow's Creek & $6.6-1.5$ & $24.5-22.9$ & $74.9-65.7$ & 153 & 6.34 & $5.8-6.0$ & + \\
5 & TVA-Gallatin & $8.2-1.6$ & $16.4-14.8$ & $74.1-62.8$ & 153 & 7.63 & $3.4-3.6$ & $\diamond$ \\
6 & TVA-Paradise & $11.3-2.3$ & $19.2-15.3$ & $102.9-71.1$ & 183 & 7.93 & $3.4-4.0$ & $\star$ \\
7 & Wildfires & $5-11$ & $22.64-40.7$ & $50-70$ & $10-68.2$ & $20-136.4$ & 1.2 & $\nabla$ \\
\hline
\end{tabular}

The data suggests that for almost all the industrial cases the plume trajectory will be virtually unaffected by the boundary layer velocity variation. In these cases (1-6) the values of $\varsigma_{m}$ and $\varsigma_{f}$ are very close to one. The only exception to this is for case 4 at lower wind speeds. In all cases the wildfire plumes are likely to be significantly altered by the boundary layer flow. The wildfire values for the transition criteria are $1.9<\varsigma_{m}<2.0$ and $54<\varsigma_{f}<101$. This was confirmed by locating the case study plumes in contour plots of $\chi=z_{45 . p} / z_{45 . u}$ as a function of $U_{0} / u_{0}$ and $z_{m} / h_{s}$ for $F r=1.2,4.0$, and 6.0. These contour plots are shown in Figures 11 to 13. In all cases the wildfire plumes were significantly influenced by the vertical variation in horizontal velocity (triangles in figure 11) whereas the vast majority of the industrial plumes had trajectories that could be well modeled by the standard Brigg's algebraic equation.

\section{Discussion and conclusions}

The trajectory of a bent-over plume in a power-law velocity profile has many applications. This problem is particularly pertinent to large, highly buoyant fire plumes that can rise high into the atmospheric boundary layer. However, the conditions under which the ambient velocity profile needs to be considered, when modeling, have not been previously investigated. The near field plume trajectory is typically quantified in terms of length scales of either momentum $\left(z_{m}\right)$ or buoyancy $\left(z_{f}\right)$. These lengths represent the vertical distance that a pure point source jet $\left(z_{m}\right)$ or plume $\left(z_{f}\right)$ would have to rise for its velocity to equal the wind speed. These lengths are smaller for plumes (forced or unforced) in a power-law boundary layer because the wind speed increases with height while the plume velocity decreases. It was shown that the trajectory of a plume in a power-law boundary layer will differ significantly from that of a plume in a uniform wind field when the boundary layer length scales are significantly smaller than the equivalent uniform velocity profile length scales. An analytic expression for the ratio of the uniform and boundary layer length scales $\left(\varsigma_{m}\right.$ and $\varsigma_{f}$ ) was derived (see Equation 23 ) and can be used to determine when it is important to model the boundary layer velocity profile and when the algebraic Briggs equation can be applied.

The results show that plumes that are released from relatively low heights ( $\operatorname{small} h_{s} / z_{m}$ of $h_{s} / z_{f}$ ) within a power-law velocity profile experience greater vertical variation in wind speed as they rise through the boundary 


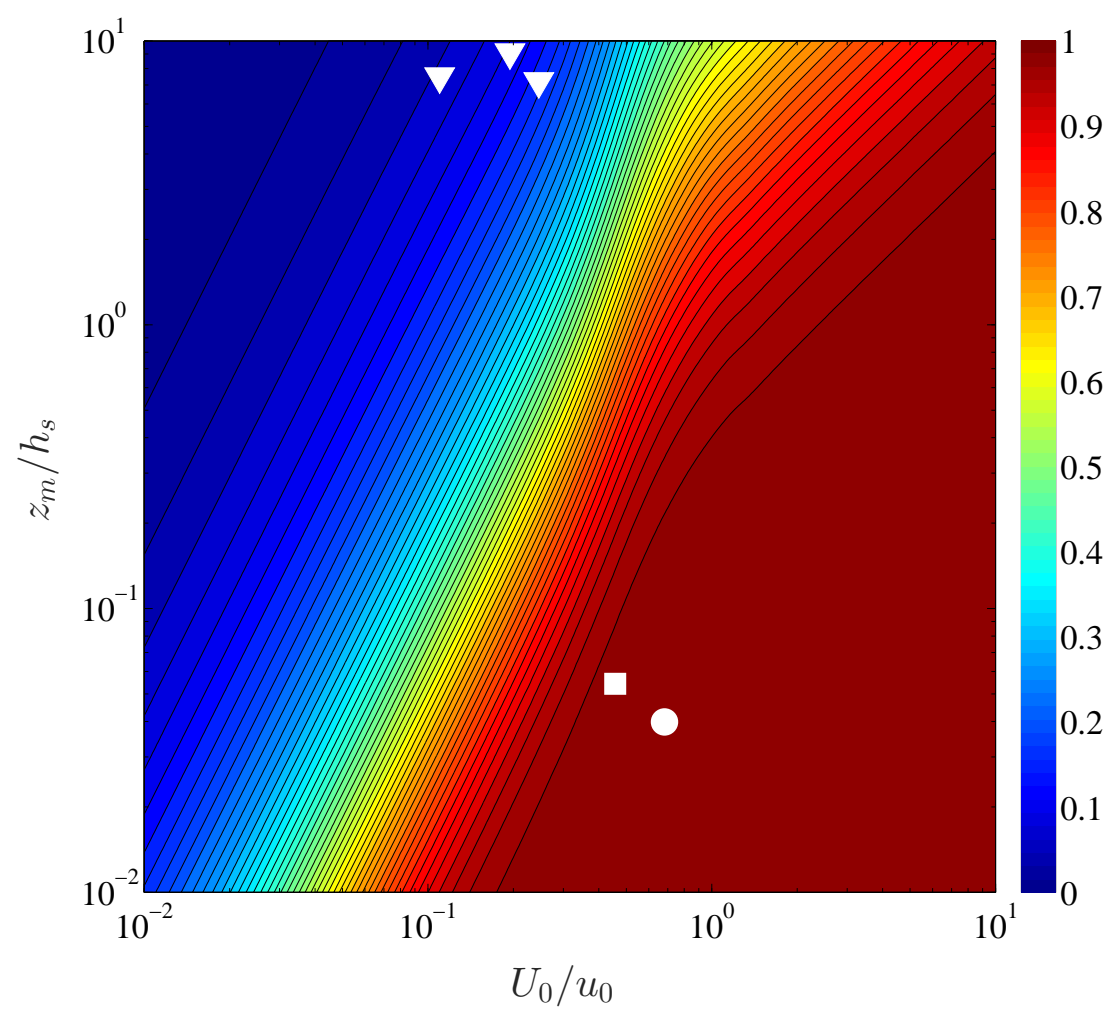

Figure 11: Contour plot of $\chi=z_{45 . p} / z_{45 . u}$ as a function of $U_{0} / u_{0}$ and $z_{m} / h_{s}$ for $F r=1.2$. Symbols are given in Table 4 .

layer, due to the increased velocity gradient near the surface. The boundary layer can also be important for plumes with initially steep trajectories (large $z_{m}$ or $z_{f}$ ). Investigation of a number of full scale industrial and wildfire plumes indicates that, in general, industrial plumes are largely unaffected by vertical variations in wind speed except for under very low wind conditions. However, wildfire plumes are significantly more buoyant than industrial plumes and have steeper trajectories that are strongly influenced by the boundary layer velocity profile.

\section{Acknowledgments}

This material is based upon work supported by the National Science Foundation under Grant No. 1200560. Any opinions, findings, and conclusions or recommendations expressed in the material are those of the authors and do not necessarily reflect the views of the NSF.

\section{References}

Abraham, G. (1963). Jet diffusion in stagnant ambient fluid. Ph.D. thesis TU Delft, Delft University of Technology. ASCE (1999). Wind tunnel studies of buildings and structures. American Society of Civil Engineers, Reston, VA, U.S.A. 


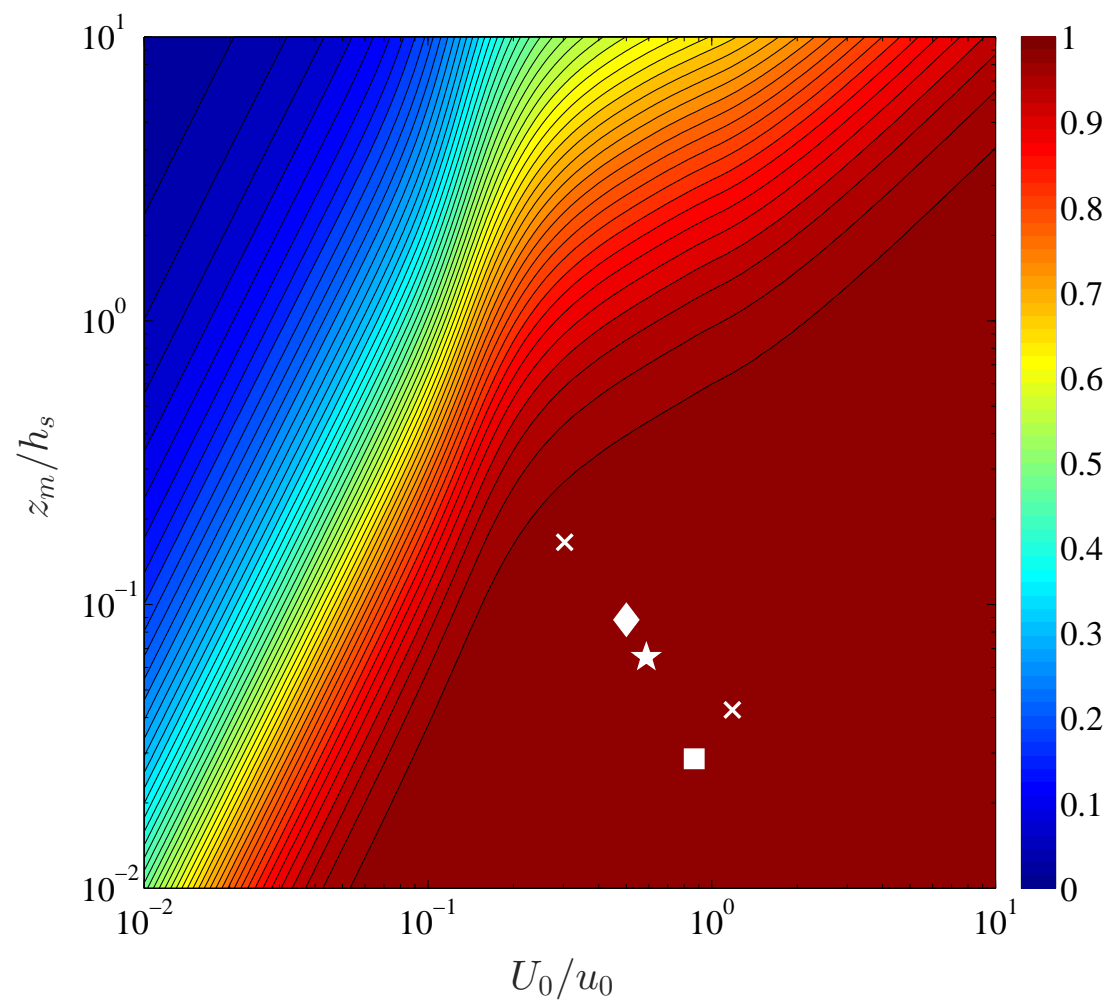

Figure 12: Contour plot of $\chi=z_{45 . p} / z_{45 . u}$ as a function of $U_{0} / u_{0}$ and $z_{m} / h_{s}$ for $F r=4.0$. Symbols are given in Table 4 .

Baines, W., \& Keffer, J. (1963). The round turbulent jet in cross wind. J Fluid Mech, 15, $481-488$.

Baum, H., \& McCaffrey, B. (1989). Fire induced flow field: theory and experiment. In Fire Safety Science: Proceedings of the Second International Symposium (pp. 129-148). Hemisphere Publishing Newport, Australia.

Bennett, M., Sutton, S., \& Gardiner, D. (1992). An analysis of lidar measurements of buoyant plume rise and dispersion at five power stations. Atmospheric Environment. Part A. General Topics, 26, 3249-3263.

Bhutia, S., Jenkins, M. A., \& Sun, R. (2010). Comparison of firebrand propagation prediction by a plume model and a coupled-fire/atmosphere large-eddy simulator. Journal of Advances in Modeling Earth Systems, 2, 4. PT: J; TC: 0; UT: WOS:000208299500004.

Briggs, G. (1975a). A comparison of the trajectories of rising buoyant plumes with theoretical empirical models. Atmospheric Environment (1967), 9, 455-457.

Briggs, G. A. (1965). A plume rise model compared with observations. Journal of the Air Pollution Control Association, 15. URL: http://dx.doi.org/10.1080/00022470.1965.10468404. doi:10.1080/00022470.1965. 10468404.

Briggs, G. A. (1968). Concawe meeting: Discussion of the comparative consequences of different plume rise formulas. Atmospheric Environment (1967), 2, 228-232. 


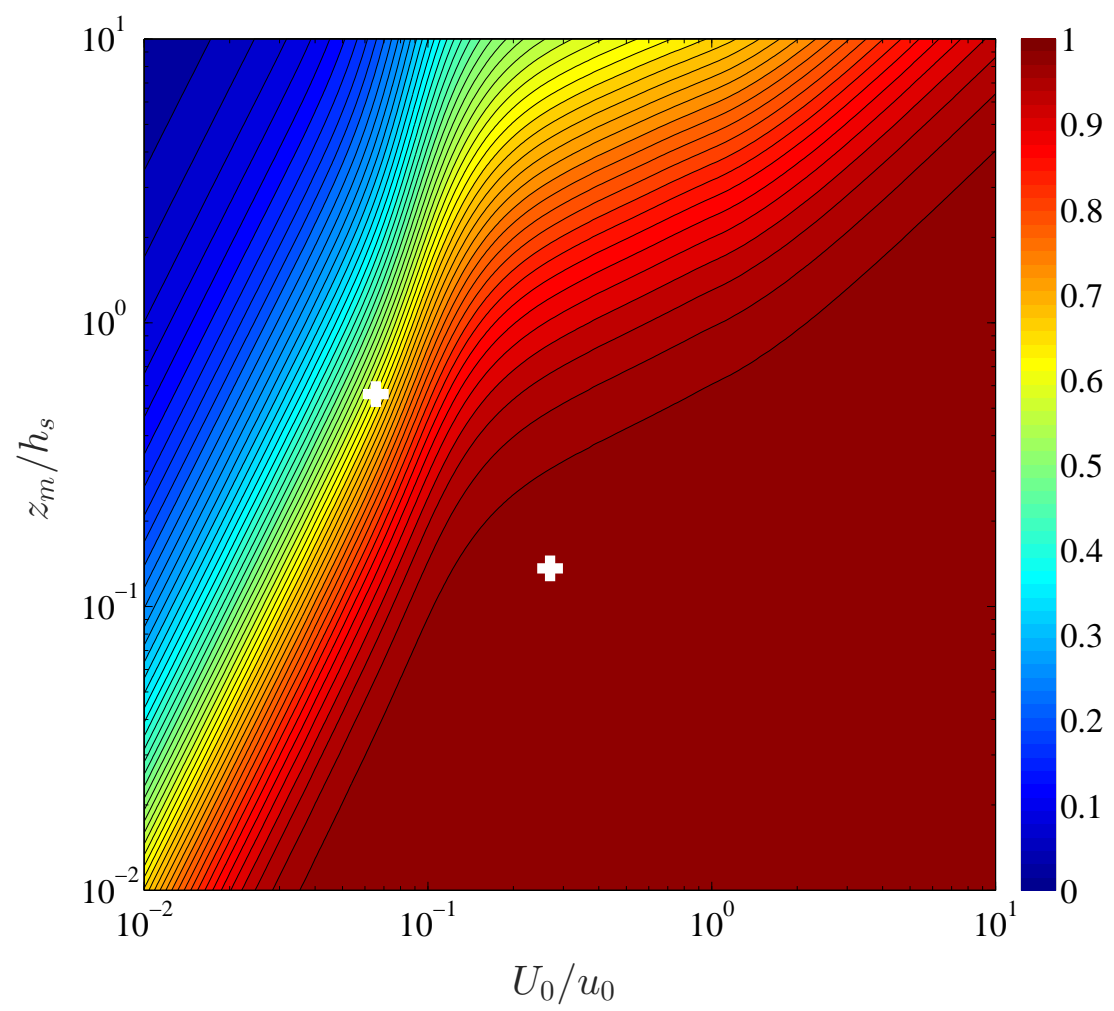

Figure 13: Contour plot of $\chi=z_{45 . p} / z_{45 . u}$ as a function of $U_{0} / u_{0}$ and $z_{m} / h_{s}$ for $F r=6.0$. Symbols are given in Table 4 .

Briggs, G. A. (1972). Chimney plumes in neutral and stable surroundings. Atmospheric Environment (1967), 6, $507-510$.

Briggs, G. A. (1975b). Plume rise predictions, lectures on air pollution and environment impact analysis. Am. Meteorol. Soc., Boston, USA, 10.

Briggs, G. A. (1984). Plume rise and buoyancy effects. Atmospheric Science and Power Production, (pp. 327-366).

Carson, J. E., \& Moses, H. (1969). The Validity of Several Plume Rise Formulas. Journal of the Air Pollution Control Association, 19, 862-866. doi:10.1080/00022470.1969.10469350.

Contini, D., Cesari, D., Donateo, A., \& Robins, A. (2009). Effects of Reynolds number on stack plume trajectories simulated with small scale models in a wind tunnel. Journal of Wind Engineering and Industrial Aerodynamics, . URL: http://dx.doi.org/10.1016/j.jweia.2009.07.007. doi:10.1016/j.jweia.2009.07.007.

Contini, D., Donateo, A., Cesari, D., \& Robins, A. G. (2011). Comparison of plume rise models against water tank experimental data for neutral and stable crossflows. Journal of Wind Engineering and Industrial Aerodynamics, $99,539-553$.

Contini, D., \& Robins, A. (2001). Water tank measurements of buoyant plume rise and structure in neutral 
crossflows. Atmospheric Environment, . URL: http://dx.doi.org/10.1016/S1352-2310(01)00398-3. doi:10. 1016/S1352-2310(01)00398-3.

Cook, N. J. (1997). The deaves and harris abl model applied to heterogeneous terrain. Journal of Wind Engineering and Industrial Aerodynamics, 66, 197-214.

Davenport, A. G. (1960). Rationale for determining design wind velocities. Technical Report DTIC Document.

Davidson, G. (1986). Gaussian versus top-hat profile assumptions in integral plume models. Atmospheric Environment (1967), 20, 471-478.

Davidson, G. (1989). Simultaneous trajectory and dilution predictions from a simple integral plume model. Atmospheric Environment, 23, 341-349.

Fischer, H. B., List, J. E., Koh, C. R., Imberger, J., \& Brooks, N. H. (2013). Mixing in inland and coastal waters. Elsevier.

Fisher, B. E. A., Metcalfe, E., Vince, I., \& Yates, A. (2001). Modelling plume rise and dispersion from pool fires. Atmospheric Environment, 35, 2101-2110.

Fric, T., \& Roshko, A. (1994). Vortical structure in the wake of a transverse jet. Journal of Fluid Mechanics, 279, $1-47$.

Frick, W. E. (1984). Non-empirical closure of the plume equations. Atmospheric Environment, 18, 653-662.

Hellmann, G. (1917). Über die Bewegung der Luft in den untersten Schichten der Atmosphäre. Königlich Preussischen Akademie der Wissenschaften.

Hoult, D. P., Fay, J. A., \& Forney, L. J. (1969). A theory of plume rise compared with field observations. Journal of the Air Pollution Control Association, 19, 585-590.

Hoult, D. P., \& Weil, J. C. (1972). Turbulent plume in a laminar cross flow. Atmospheric Environment (1967), 6, $513-531$.

Hübner, J. (2004). Buoyant plumes in a turbulent environment. Ph.D. thesis University of Cambridge.

Hunt, G. R., \& Kaye, N. B. (2005). Lazy plumes. Journal of Fluid Mechanics, 533, 329-338. URL: http: //dx.doi.org/10.1017/S002211200500457X.

Jordinson, R. (1956). Flow in a jet directed normal to the wind. R. \& $M$, .

Kaye, N. B. (2008). Turbulent plumes in stratified environments: A review of recent work. Atmosphere-Ocean, 46 , 433-441.

Kaye, N. B., \& Hunt, G. R. (2009). An experimental study of large area source turbulent plumes. International Journal of Heat and Fluid Flow, 30, 1099-105. URL: http://dx.doi.org/10.1016/j . ijheatfluidflow. 2009. 05.001. 
Lee, J. H.-w., \& Chu, V. (2003). Turbulent jets and plumes: a Lagrangian approach. Springer Science \& Business Media

Li, Q., Zhi, L., \& Hu, F. (2010). Boundary layer wind structure from observations on a 325m tower. Journal of wind engineering and industrial aerodynamics, 98, 818-832.

Linden, P. F., \& Kaye, N. B. (2006). Interacting turbulent plumes in a naturally ventilated enclosure. Int. J. of Vent., 4, 301-310.

Marro, M., Salizzoni, P., Cierco, F., Korsakissok, I., Danzi, E., \& Soulhac, L. (2014). Plume rise and spread in buoyant releases from elevated sources in the lower atmosphere. Environmental Fluid Mechanics, 14, 201-219.

Mercer, G., \& Weber, R. (1994). Plumes above line fires in a cross-wind. International Journal of Wildland Fire, 4, 201-207.

Morton, B., \& Middleton, J. (1973). Scale diagrams for forced plumes. Journal of Fluid Mechanics, 58, 165-176.

Morton, B., Taylor, G., \& Turner, J. (1956). Turbulent gravitational convection from maintained and instantaneous sources, . 234, 1-23.

Morton, B. R. (1965). Modeling fire plumes. Symposium (International) on Combustion, 10, 973-982.

Ooms, G., \& Mahieu, A. (1981). A comparison between a plume path model and a virtual point source model for a stack plume. Applied Scientific Research, 36, 339-356.

Priestly, C. H. B., \& Ball, F. K. (1955). Continuous convection from an isolated source of heat, . 81, 144-157.

Sardoy, N., Consalvi, J.-L., Porterie, B., \& Fernandez-Pello, A. (2007). Modeling transport and combustion of firebrands from burning trees. Combustion and Flame, 150, 151-169. URL: http://dx.doi.org/10.1016/j . combustflame.2007.04.008.

Scase, M. M., Caulfield, C. P., Dalziel, S. B., \& Hunt, J. C. R. (2006). Time-dependent plumes and jets with decreasing source strengths, . 563, 443-461.

Schatzman, M., \& Policastro Anthony J. (1984). Plume rise from stacks with scrubbers: A state of the art review. Bulletin of the American Meteorological Society, 65, 210-215.

Smith, S. H., \& Mungal, M. G. (1998). Mixing, structure and scaling of the jet in crossflow. J. Fluid Mech., 357, $83-122$.

Thurston, W., Fawcett, R. J., Tory, K. J., \& Kepert, J. D. (2015). Simulating boundary-layer rolls with a numerical weather prediction model. Quarterly Journal of the Royal Meteorological Society, .

Tieleman, H. W. (2008). Strong wind observations in the atmospheric surface layer. Journal of Wind Engineering and Industrial Aerodynamics, 96, 41-77.

Turner, J. S. (1973). Buoyancy effects in fluids. CUP. 

Wang, H., \& Law, A. W.-K. (2002). Second-order integral model for a round turbulent buoyant jet, . 459, 397-428.

${ }_{377}$ Weil, J. (1988). Plume rise. Lectures on air pollution modeling, (pp. 119-166).

378 Zonato, C., Vidili, A., Pastorino, R., \& De Faveri, D. (1993). Plume rise of smoke coming from free burning fires. 379 Journal of Hazardous Materials, 34, 69-79. 


\section{Appendix A. Plume Fluxes Equations}

A control volume analysis is presented to derive a set of ordinary differential equations for the plume properties as a function of distance along the plume centerline. As the negligible role of turbulence is discussed in Sect. 1, the model is thus derived for steady smooth cross-flow with arbitrary velocity profile. The concept of local similarity is extended to the bent-over plume in accordance with Baines \& Keffer (1963). As a result of the local similarity assumption and the aforementioned study conducted by Davidson (1986), the velocity and density defect profiles are considered to be top-hat. In contrast to previous studies (Briggs, 1968, 1972, 1975b,a; Hoult et al., 1969; Hoult \& Weil, 1972; Davidson, 1989; Mercer \& Weber, 1994) the plume cross section normal to the plume centerline trajectory is taken to be an ellipse. See Figure (A.14). The ratio of major to conjugate radii is denoted by $\lambda$.

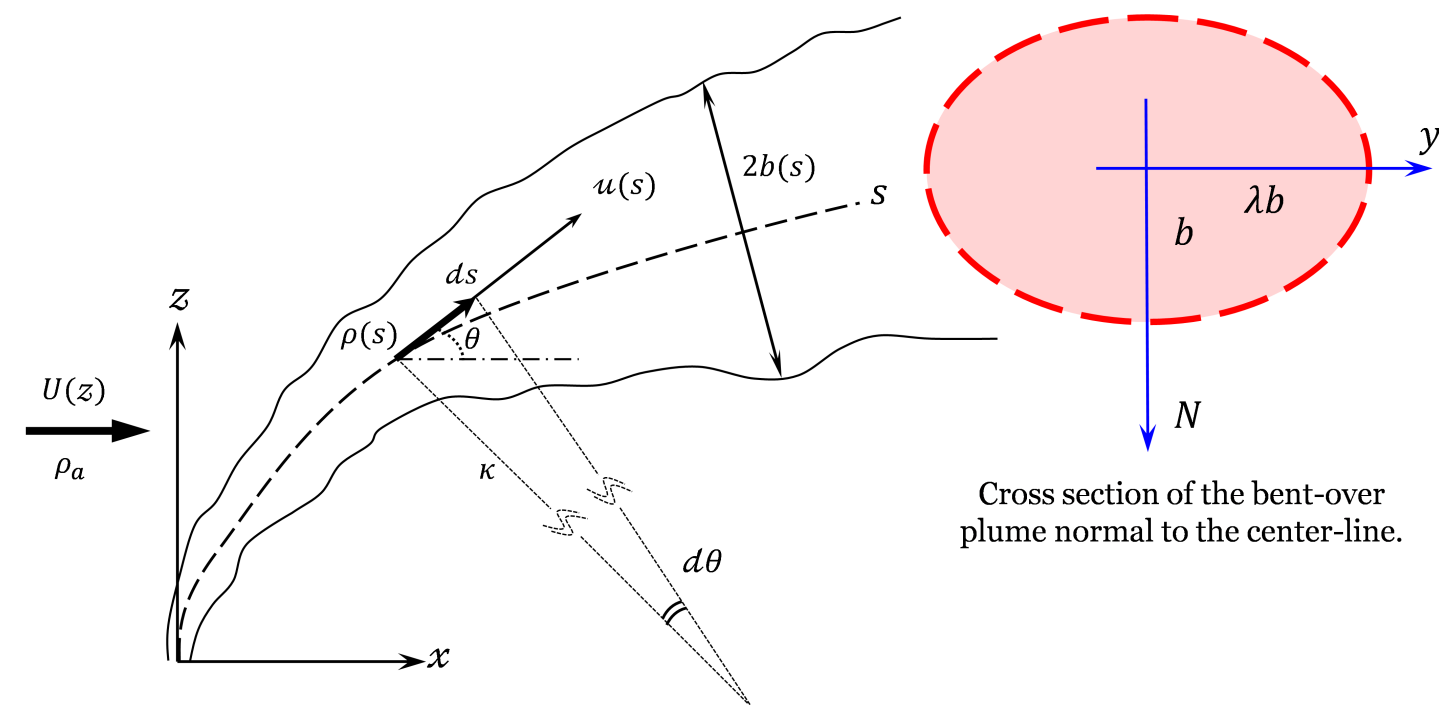

Figure A.14: (Right) Coordinate system for a bent-over buoyant plume through a cross flow; (Left) Normal cross section area of the plume perpendicular to the centerline $s$.

Based on laboratory observations of Jordinson (1956) it is known that the main factor in determining the trajectory of a bent-over plume is the rate at which it entrains mass from the ambient environment. According to the observations of Hoult et al. (1969), there are two entrainment mechanisms involved. One is due to the difference between the plume velocity along $s$, i.e. $u(s)$, and the wind velocity component parallel to the plume center-line, that is longitudinal shear driven entrainment. The other is due to the velocity component of the wind normal to the plume envelope, i.e. $U \sin \theta$; see Figure (A.15b). The two mechanisms are taken to be additive such that the entrainment velocity, $u_{e}$, can be written as:

$$
u_{e}=\alpha|u-U \cos (\theta)|+\beta|U \sin (\theta)|
$$

where $\alpha$ is entrainment coefficient for longitudinal shear driven mixing and $\beta$ is the transverse entrainment coefficient that parameterizes entrainment due to transverse shear. It is assumed that both coefficients are independent of the position along the plume trajectory. 
The plume trajectory's governing equations can be derived from conservation of mass, momentum, and energy. Pursuant to Figure (A.15a), for an infinitesimally thin plume cross section normal to the plume trajectory, the continuity equation reads:
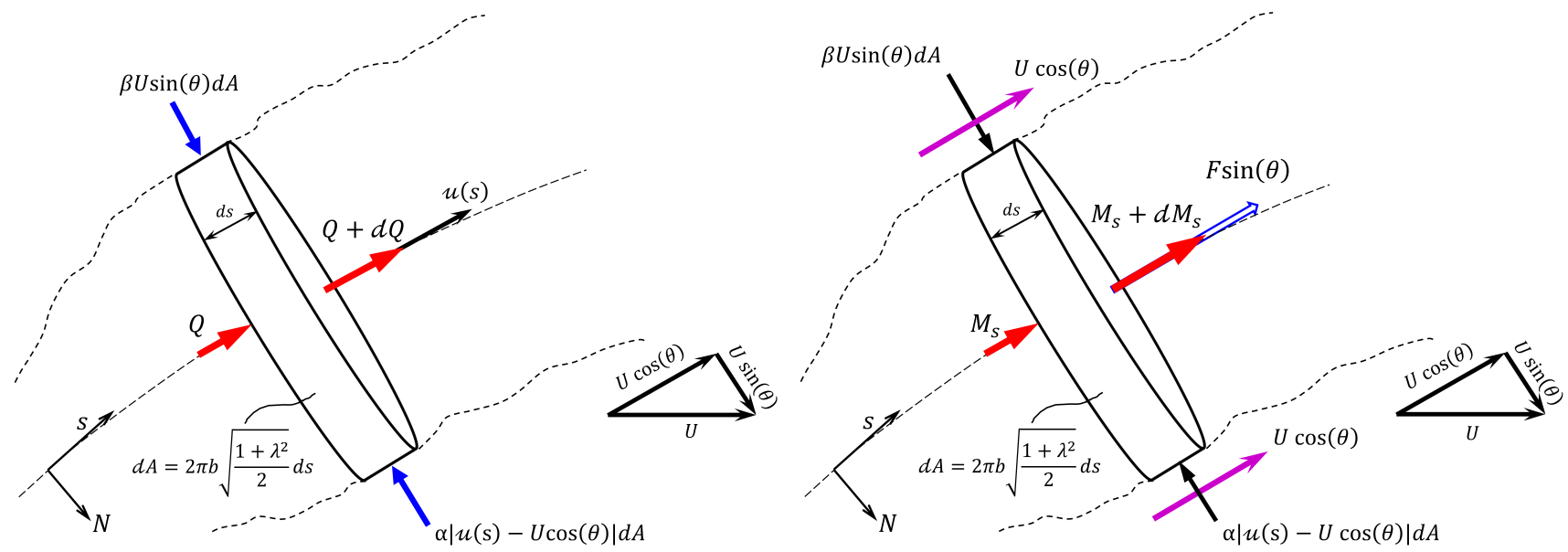

(a) Mass conservation diagram.

(b) Momentum diagram along $s$.

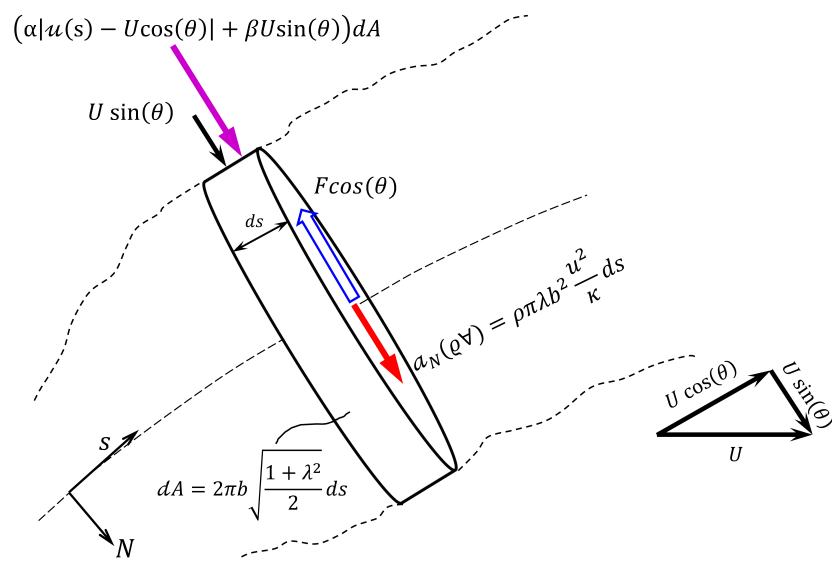

(c) Momentum diagram along $N$.

(d) Buoyancy flux diagram.

Figure A.15: Schematic of an infinitesimal bent-over plume element. Net buoyancy force, that is $\left(\varrho_{a}-\varrho\right) g \pi \lambda b^{2} d s$, is denoted by $F$. Through above diagrams, sharp head arrows denote velocity vectors, bold head arrows denote flux vectors, and hollow arrows denote force vectors.

$$
\frac{d(\varrho \forall)}{d t}+\oiiint_{A_{e}} \varrho \bar{u} \cdot \bar{n} d A_{e}=0
$$

where $\varrho \forall$ is the control volume mass in which $\forall$ denotes volume of the plume element, $A_{e}$ is the peripheral surface area of the plume element and $\bar{n}$ is the outward normal vector to $A_{e}$. Expanding the integral in the $s$ direction leads to:

$$
\begin{aligned}
\pi \varrho \lambda b^{2} u+\frac{d}{d s}\left(\pi \varrho \lambda b^{2} u\right) d s-\pi \varrho \lambda b^{2} u= & 2 \pi b \sqrt{\frac{1+\lambda^{2}}{2}} \varrho_{a} \beta U \sin (\theta) d s+ \\
& 2 \pi b \sqrt{\frac{1+\lambda^{2}}{2}} \varrho_{a} \alpha|u-U \cos (\theta)| d s .
\end{aligned}
$$


Simplifying equation (A.3) gives conservation of mass along the plume trajectory:

$$
\frac{d}{d s}\left(\pi \varrho \lambda b^{2} u\right)=2 \pi b \sqrt{\frac{1+\lambda^{2}}{2}} \varrho_{a}(\beta U \sin (\theta)+\alpha|u-U \cos (\theta)|) .
$$

The momentum equation in the $s$ direction is a balance of acceleration, entrianed momentum and buoyancy:

$$
\tau_{s}=\left(\oiiint_{A_{e}} \varrho \bar{u} \cdot \bar{n} \bar{u} d A_{e}\right)_{s}
$$

where $\tau_{s}$ denotes the resultant of the external forces acting in $s$ direction on the control volume. Considering Figure (A.15b), applying equation (A.5), and expanding its terms yields:

$$
\begin{array}{r}
\left(\varrho_{a}-\varrho\right) g\left(\pi \lambda b^{2} d s\right) \sin (\theta)=\left(\pi \varrho \lambda b^{2} u^{2}\right)+\frac{d}{d s}\left(\pi \varrho \lambda b^{2} u^{2}\right) d s-\left(\pi \varrho \lambda b^{2} u^{2}\right)- \\
{\left[2 \pi b \sqrt{\frac{1+\lambda^{2}}{2}} \varrho_{a}(\beta U \sin (\theta)+\alpha|u-U \cos (\theta)|)\right] U \cos (\theta) d s}
\end{array}
$$

Using the continuity equation, momentum conservation in the $s$ direction is obtained by simplifying Equation (A.6):

$$
\frac{d}{d s}\left(\pi \varrho \lambda b^{2} u^{2}\right)-U \cos (\theta) \frac{d}{d s}\left(\pi \varrho \lambda b^{2} u\right)=\left(\varrho_{a}-\varrho\right) g \pi \lambda b^{2} \sin (\theta)
$$

Similarly, the transverse momentum equation is a balance between the centripetal acceleration, the entrained momentum, and buoyancy:

$$
\tau_{N}=\left(\oiint_{A_{e}} \varrho \bar{u} \cdot \bar{n} \bar{u} d A_{e}\right)_{N}
$$

in which, $\tau_{n}$ denotes the resultant force of the external forces acting in the transverse direction. Expanding each term based on Figure (A.15c) leads to:

$$
-\left(\varrho_{a}-\varrho\right) g\left(\pi \lambda b^{2} d s\right) \cos (\theta)=\left(\varrho \pi \lambda b^{2} d s \frac{u^{2}}{\kappa}\right)-\left[2 \pi b \sqrt{\frac{1+\lambda^{2}}{2}} \varrho_{a}(\beta U \sin (\theta)+\alpha|u-U \cos (\theta)|) d s\right] U \sin (\theta)
$$

where $\kappa$ is the radius of the plume center-line's curvature. From geometrical considerations,$\kappa=-d s / d \theta$. The transverse conservation of momentum equation is derived by substituting $\kappa$ into (A.9) and simplifying it using the conservation of mass equation to get

$$
\left(\pi \varrho \lambda b^{2} u^{2}\right) \frac{d \theta}{d s}+U \sin \theta \frac{d}{d s}\left(\pi \varrho \lambda b^{2} u\right)=\left(\varrho_{a}-\varrho\right) g\left(\pi \lambda b^{2}\right) \cos (\theta)
$$

It is assumed that all temperature differences are small, which leads to limited density defect ratios, except regions adjacent to flames (near field) (see Schatzman \& Policastro Anthony J. (1984)). As a result, it is possible to use the Boussinesq approximation. Since temperature differences are small and the ambient fluid would predominantly be in gaseous phase, temperature localization is allowed and equation of excess energy can be written as buoyancy conservation

$$
\iiint_{\forall} \Delta \varrho g \nabla \cdot \bar{u} d \forall=F_{\text {potential }} .
$$


In the above expression $\Delta \varrho$ is the density defect along the plume centerline and $F_{\text {potential }}$ is the potential buoyancy that the ambient environment can provide due to the temperature differences inside the plume envelope. From Figure (A.15d) and (A.11) one can write:

$$
\Delta \varrho g\left(\pi \lambda b^{2} u\right)+\frac{d}{d s}\left(\Delta \varrho g \pi \lambda b^{2} u\right) d s-\Delta \varrho g \pi \lambda b^{2} u=\left(\varrho_{a}-\varrho\right) g \pi \lambda b^{2} u
$$

which can be simplified to

$$
\frac{d}{d s}\left(\Delta \varrho g \pi \lambda b^{2} u\right)=g \pi \lambda b^{2} u \frac{d \varrho}{d s}
$$

in which $d \varrho=\left(\varrho_{a}-\varrho\right)$. Substitution of $d s=d z / \sin (\theta)$ into (A.13) gives the expression for conservation of excess energy (buoyancy) along the plume trajectory:

$$
\frac{d}{d s}\left(\Delta \varrho g \pi \lambda b^{2} u\right)=g \pi \lambda b^{2} u \frac{d \varrho}{d z} \sin (\theta) .
$$

Finally the plume rise height $(z)$ and downstream distance $(x)$ of the plume are obtained by integrating along the plume trajectory centerline:

$$
\begin{aligned}
& x=\int_{0}^{s} \cos (\theta) d s \\
& z=\int_{0}^{s} \sin (\theta) d s
\end{aligned}
$$

Thus far, (A.4), (A.7), (A.10), (A.14), (A.15) and (A.16) constitute a set of fully-coupled differential algebraic equations involving the plume velocity, buoyancy, radius, and trajectory angle. In order to simplify the set of DAEs the equations are rewritten in terms of a new set of physically meaningful dependent variables as follows:

$$
\begin{aligned}
Q & =\lambda b^{2} u \\
M & =\lambda b^{2} u^{2} \\
F & =g^{\prime} \lambda b^{2} u \\
u & =M / Q \\
b & =Q / \sqrt{\lambda M}
\end{aligned}
$$

where $Q$ is specific flow rate, $M$ is specific momentum flux, $g^{\prime}$ is reduced gravity acceleration and specific buoyancy flux is denoted by $F$. The final set of dimensional equations are rewritten in terms of specific fluxes as

$$
\begin{gathered}
\frac{d Q}{d s}=Q \sqrt{\frac{2\left(1+\lambda^{2}\right)}{M \lambda}}\left(\alpha\left|\frac{M}{Q}-U \cos (\theta)\right|+\beta|U \sin (\theta)|\right), \\
\frac{d M}{d s}-U \cos (\theta) \frac{d Q}{d s}=\frac{F Q}{M} \sin (\theta) \\
U \sin (\theta) \frac{d Q}{d s}+M \frac{d \theta}{d s}=\frac{F Q}{M} \cos (\theta)
\end{gathered}
$$

and

$$
\frac{d F}{d s}=Q \frac{g}{\varrho_{a}} \frac{d \varrho}{d z} \sin (\theta)=-Q \mathrm{~N}^{2} \sin (\theta)
$$

where $\mathrm{N}_{\mathrm{N}}$ is the Brunt-Vaisala frequency. 